\title{
LINE ITERATIVE METHODS FOR CYCLICALLY REDUCED DISCRETE CONVECTION-DIFFUSION PROBLEMS*
}

\author{
HOWARD C. ELMAN ${ }^{\dagger}$ AND GENE H. GOLUB ${ }^{\ddagger}$
}

\begin{abstract}
An analytic and empirical study of line iterative methods for solving the discrete convection-diffusion equation is performed. The methodology consists of performing one step of the cyclic reduction method, followed by iteration on the resulting reduced system using line orderings of the reduced grid. Two classes of iterative methods are considered: block stationary methods, such as the block Gauss-Seidel and SOR methods, and preconditioned generalized minimum residual methods with incomplete LU preconditioners. New analysis extends convergence bounds for constant coefficient problems to problems with separable variable coefficients. In addition, analytic results show that iterative methods based on incomplete LU preconditioners have faster convergence rates than block Jacobi relaxation methods. Numerical experiments examine additional properties of the two classes of methods, including the effects of direction of flow, discretization, and grid ordering on performance.
\end{abstract}

Key words. iterative methods, line orderings, reduced system, convection-diffusion, elliptic operators

AMS(MOS) subject classifications. primary $65 \mathrm{~F} 10,65 \mathrm{~N} 20$; secondary $15 \mathrm{~A} 06$

1. Introduction. Consider the convection-diffusion equation

$$
\begin{gathered}
-\left[\left(p u_{x}\right)_{x}+\left(q u_{y}\right)_{y}\right]+r u_{x}+s u_{y}=f \quad \text { on } \Omega \\
\alpha u+\beta u_{n}=g \quad \text { on } \partial \Omega,
\end{gathered}
$$

where $\Omega$ is a smooth domain in $\mathbf{R}^{2}$ and $p>0, q>0$ on $\Omega$. Discretization of (1.1) produces a linear system of equations

$$
A u=f
$$

where $u$ and $f$ are now vectors in a finite-dimensional space, and $A$ is a nonsymmetric matrix when $r$ and $s$ are nonzero. We are concerned with discretizations (principally, finite difference methods) for which each equation in (1.2) is centered at some mesh point $\left(x_{i}, y_{j}\right)$, and the associated unknown $u_{i j}$ depends only on its neighbors in the horizontal and vertical directions. That is, the equation centered at $\left(x_{i}, y_{j}\right)$ has the form

$$
a_{i j} u_{i j}=f_{i j}-b_{i j} u_{i, j-1}-c_{i j} u_{i-1, j}-d_{i j} u_{i+1, j}-e_{i j} u_{i, j+1} \text {. }
$$

In this case, we say that (1.2) has a computational molecule of the form

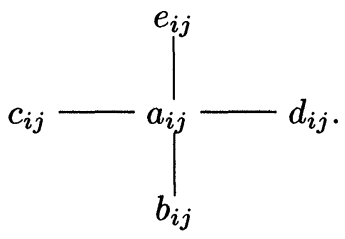

* Received by the editors April 5, 1990; accepted for publication (in revised form) November 26, 1990.

$\dagger$ Department of Computer Science, and Institute for Advanced Computer Studies, University of Maryland, College Park, Maryland 20742. The work of this author was supported by National Science Foundation grants DMS-8607478 and ASC-8958544, and by U. S. Army Research Office grant DAAL-0389-K-0016.

$\ddagger$ Department of Computer Science, Stanford University, Stanford, California 94305. The work of this author was supported by National Science Foundation grant DCR-8412314. 


$\left.\begin{array}{|llllll}\hline \otimes_{15} & \times_{25} & \otimes_{35} & \times_{45} & \otimes_{55} & \times_{65} \\ \times_{14} & \otimes_{24} & \times_{34} & \otimes_{44} & \times_{54} & \otimes_{64} \\ \otimes_{13} & \times_{23} & \otimes_{33} & \times_{43} & \otimes_{53} & \times_{63} \\ \times_{12} & \otimes_{22} & \times_{32} & \otimes_{42} & \times_{52} & \otimes_{62} \\ \otimes_{11} & \times_{21} & \otimes_{31} & \times_{41} & \otimes_{51} & \times_{61}\end{array}\right]\left[\begin{array}{cccccc}\otimes_{13} & \times_{28} & \otimes_{14} & \times_{29} & \otimes_{15} & \times_{30} \\ \times_{25} & \otimes_{10} & \times_{26} & \otimes_{11} & \times_{27} & \otimes_{12} \\ \otimes_{7} & \times_{22} & \otimes_{8} & \times_{23} & \otimes_{9} & \times_{24} \\ \times_{19} & \otimes_{4} & \times_{20} & \otimes_{5} & \times_{21} & \otimes_{6} \\ \otimes_{1} & \times_{16} & \otimes_{2} & \times_{17} & \otimes_{3} & \times_{18} \\ \hline\end{array}\right.$

FIG. 1.1. A $6 \times 5$ grid and a red-black ordering. Grid indices are shown on the left, and vector indices for a red-black ordering are shown on the right. Red points are denoted by " $\otimes$ " and black points by " $\times . "$

When the system (1.2) has this property, the mesh points $\left\{\left(x_{i}, y_{j}\right)\right\}$ and unknowns $\left\{u_{i j}\right\}$ can be ordered with a red-black ordering so that every equation centered at a "red" point depends only on "black" unknowns, and every equation centered at a "black" point depends only on "red" unknowns. An example of a red-black ordering of a $6 \times 5$ grid is shown in Fig. 1.1. If $u_{i j}$ is a black unknown, then by adding appropriate linear combinations of the equations for $u_{i \pm 1, j}$ and $u_{i, j \pm 1}$ to the equation for $u_{i j}$, we can eliminate the dependence of $u_{i j}$ on its red neighbors. When this is done for every black equation, the result is a smaller linear system

$$
A^{(b)} u^{(b)}=g^{(b)},
$$

where $u^{(b)}$ is the set of unknowns associated with black mesh points. In matrix notation, this process corresponds to ordering the rows and columns of $A$ so that (1.2) has the form

$$
\left(\begin{array}{ll}
D & C \\
E & F
\end{array}\right)\left(\begin{array}{l}
u^{(r)} \\
u^{(b)}
\end{array}\right)=\left(\begin{array}{l}
f^{(r)} \\
f^{(b)}
\end{array}\right)
$$

where $D$ and $F$ are nonsingular diagonal matrices. Decoupling of the red points $u^{(r)}$ is equivalent to producing the system (1.4), where $A^{(b)}=F-E D^{-1} C$ and $g^{(b)}=f^{(b)}-E D^{-1} f^{(r)}$.

In [7], [8], we analyzed the convergence behavior of block iterative methods for solving the reduced system (1.4) derived from discretizations of (1.1). We considered block Jacobi, Gauss-Seidel, and successive overrelaxation (SOR) methods [25], [28], where the blockings (of the rows and columns of $A^{(b)}$ ) are derived from certain line orderings of the underlying reduced (black) grid. In particular, the unknown grid values $u^{(b)}$ can be grouped together either by individual lines of the grid, producing a class of one-line orderings, or by pairs of lines, producing two-line orderings (see $\S 2$ ). These orderings produce matrices with block Property A, so that the classical analysis of Gauss-Seidel and SOR methods [25], [28] can be used. The results of [7], [8] apply to problems with the constant coefficients $p(x, y)=q(x, y)=1, r(x, y)=$ $\sigma, s(x, y)=\tau$. They show that convergence is often very fast; in particular, for nonselfadjoint problems ( $\sigma$ or $\tau$ nonzero), convergence is typically faster than for selfadjoint problems. They also show that convergence rates for solving the reduced system are often faster than for solving the full system (1.2) by analogous line methods. These observations are in agreement with asymptotic results in [20] and the algebraic analysis of [13]. Related results for point iterative methods are given in [18].

In this paper, we extend the analysis of [7], [8] to separable problems, and we also use it to derive bounds on convergence behavior for stationary methods based on incomplete factorizations [17]. In addition, in a series of numerical experiments, we examine the effect of physically significant properties of the problem (1.1) on the 
performance of iterative methods applied to (1.4). Here, we consider both block relaxation methods and the preconditioned generalized minimum residual method (GMRES) [23], with preconditioning by incomplete factorizations [17]. We focus on the following issues:

1. For constant coefficient problems, the effect of the signs and magnitudes of $r$ and $s$ in (1.1). These quantities determine the direction and rate of flow associated with the convection in the model. The analysis of [7], [8] is sensitive to magnitudes but not to signs.

2. The effect of variable coefficients $r$ and $s$. We consider problems both with and without turning points.

3. The effects of the choice of discretization on performance; we consider centered and upwind finite difference discretizations.

4. The first three issues do not address the issue of accuracy of the discrete solution. We also examine the effect of methods designed to improve accuracy in the presence of boundary layers, in particular, local mesh refinement and defect correction methods [12], [15].

An outline of the paper is as follows. In $\S 2$, we describe the reduced matrix $A^{(b)}$, and we present the ordering strategies and iterative methods used to solve (1.4), including some block red-black strategies of use for vector and parallel computations. In $\S 3$, we extend the analysis of [7], [8] to separable problems and incomplete factorizations. In $\S 4$, we describe the results of numerical experiments with constant coefficient problems. For several ordering strategies, we examine how performances of block stationary methods and preconditioned GMRES are affected by direction and rate of flow, choice of difference scheme, and use of local mesh refinement to resolve boundary layers. In $\S 5$, we compare experimental results with analytic bounds on convergence, for separable problems. In $\S 6$, we consider performance for some problems with nonseparable variable coefficients, i.e., where the flow varies in both direction and magnitude in $\Omega$. Here we consider both centered and upwind finite differences, as well as a difference scheme used to implement defect correction methods. Finally, in $\S 7$ we make some concluding remarks.

2. The reduced system and line iterative methods. Let $u_{i j}$ be a black point not next to the boundary $\partial \Omega$. Elimination of the unknowns $u_{i \pm 1, j}$ and $u_{i, j \pm 1}$ from (1.3) produces an equation in the reduced system with the computational molecule shown in Fig. 2.1. The value "*" in the center is

$$
a_{i j}-\frac{b_{i j} e_{i, j-1}}{a_{i, j-1}}-\frac{c_{i j} d_{i-1, j}}{a_{i-1, j}}-\frac{d_{i j} c_{i+1, j}}{a_{i+1, j}}-\frac{e_{i j} b_{i, j+1}}{a_{i, j+1}},
$$

and the right-hand side is perturbed by an average of neighboring values,

$$
g_{i j}^{(b)}=f_{i j}-\frac{b_{i j} f_{i, j-1}}{a_{i, j-1}}-\frac{c_{i j} f_{i-1, j}}{a_{i-1, j}}-\frac{d_{i j} f_{i+1, j}}{a_{i+1, j}}-\frac{e_{i j} f_{i, j+1}}{a_{i, j+1}} .
$$

The line ordering strategies for the reduced grid are outlined as follows (see [7], [8] for further details). In the natural one-line ordering, points of the reduced grid are grouped together by diagonal lines, e.g., oriented in the NW-SE direction. The left side of Fig. 2.2 shows an example for a $6 \times 5$ grid. Here, the $k$ th line consists of all points with grid indices $(i, j)$ such that $i+j=2 k+1$. (Compare with the left side of Fig. 1.1.) Thus, in Fig. 2.2, the first line consists of the points $\{1,2\}$, the second line consists of the points $\{3,4,5,6\}$, etc. In the natural two-line ordering, points 


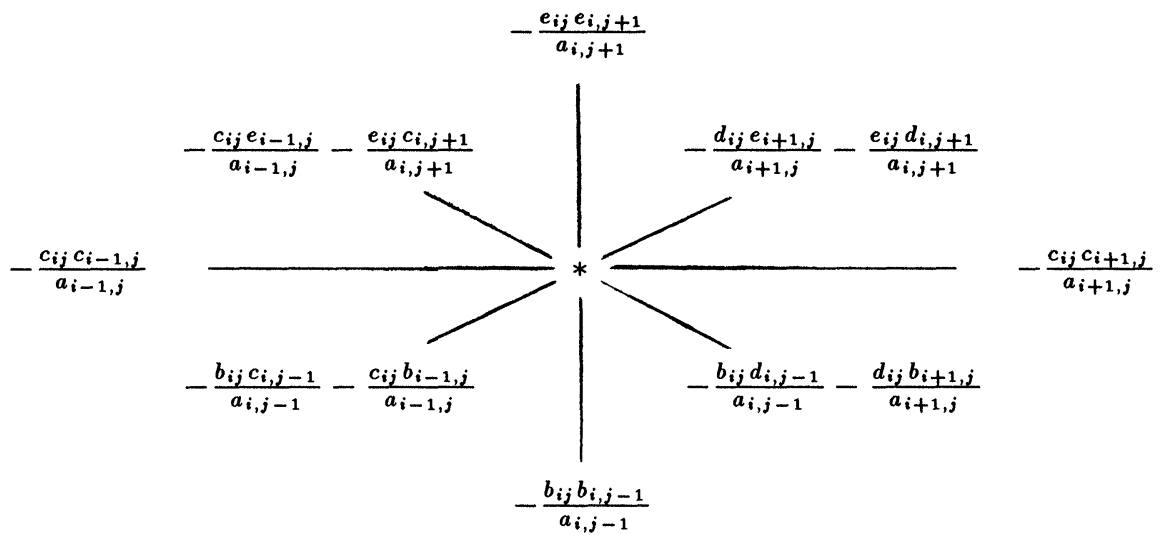

FiG. 2.1. The computational molecule for the reduced system.

\begin{tabular}{|cccccc|}
\hline$\cdot$ & $\times_{11}$ & $\cdot$ & $\times_{14}$ & $\cdot$ & $\times_{15}$ \\
$\times_{6}$ & $\cdot$ & $\times_{10}$ & $\cdot$ & $\times_{13}$ & $\cdot$ \\
$\cdot$ & $\times_{5}$ & $\cdot$ & $\times_{9}$ & $\cdot$ & $\times_{12}$ \\
$\times_{2}$ & $\cdot$ & $\times_{4}$ & $\cdot$ & $\times_{8}$ & $\cdot$ \\
$\cdot$ & $\times_{1}$ & $\cdot$ & $\times_{3}$ & $\cdot$ & $\times_{7}$ \\
\hline
\end{tabular}

\begin{tabular}{|cccccc|}
\hline$\cdot$ & $\times_{13}$ & $\cdot$ & $\times_{14}$ & $\cdot$ & $\times_{15}$ \\
$\times_{7}$ & $\cdot$ & $\times_{9}$ & $\cdot$ & $\times_{11}$ & $\cdot$ \\
$\cdot$ & $\times_{8}$ & $\cdot$ & $\times_{10}$ & $\cdot$ & $\times_{12}$ \\
$\times_{1}$ & $\cdot$ & $\times_{3}$ & $\cdot$ & $\times_{5}$ & $\cdot$ \\
$\cdot$ & $\times_{2}$ & $\cdot$ & $\times_{4}$ & $\cdot$ & $\times_{6}$ \\
\hline
\end{tabular}

Fig. 2.2. Natural one-line (left) and two-line (right) orderings of the reduced $6 \times 5$ grid.

are grouped together by pairs of either horizontal or vertical lines. The right side of Fig. 2.2 shows an example of a horizontal grouping for a $6 \times 5$ grid. The points in the $k$ th group are those with grid indices $(i, j)$ such that $k-1<j / 2 \leq k$. If the number of lines is odd, the last group consists of a single line, as in the group $\{13,14,15\}$. For both these strategies, $A^{(b)}$ is a block tridiagonal matrix; let $D$ denote its block diagonal. For the one-line ordering, each block of $D$ is a tridiagonal matrix, and for the two-line ordering, each block of $D$ is a pentadiagonal matrix (except possibly the last block, which may be tridiagonal). It is also useful (e.g., for parallel computations, see [8]) to define line red-black variants of these orderings, in which alternating lines (or line pairs) are assigned opposite colors. For example, for the one-line version, let the sets $\{1,2\},\{7,8,9,10,11\}$, and $\{15\}$ be denoted as "red" lines, and the others as "black" lines. Then every equation centered at a point in a red line depends only on that red line and the neighboring black lines; an analogous statement holds for equations centered on black lines. For the red-black one-line ordering, all red lines are ordered first, followed by all black lines. The red-black two-line ordering is defined in similar fashion.

For any of these line orderings, let

$$
A^{(b)}=D-C=(D-L)-U
$$

where $D$ is the block diagonal part of $A^{(b)}$ and $L$ and $U$ are the lower and upper triangular parts, respectively, of the block off-diagonal part of $A^{(b)}$. We consider several block stationary methods based on the splittings (2.1). The block Jacobi 
iteration is given by

$$
u_{k+1}^{(b)}=D^{-1} C u_{k}^{(b)}+D^{-1} g^{(b)}
$$

and the block SOR iteration is

$$
u_{k+1}^{(b)}=(D-\omega L)^{-1}[(1-\omega) D+\omega U] u_{k}^{(b)}+\omega(D-\omega L)^{-1} g^{(b)} .
$$

The block Gauss-Seidel iteration corresponds to the case $\omega=1$ in 2.2. In all cases, $A^{(b)}$ is block consistently ordered, so that [28]

$$
\rho\left((D-L)^{-1} U\right)=\left[\rho\left(D^{-1} C\right)\right]^{2},
$$

where $\rho(X)$ denotes the spectral radius of a matrix $X$.

In addition, we consider the use of the ILU(0) incomplete factorization [17] applied to $A^{(b)}$ for each of the orderings. This factorization is defined as

$$
M=(\hat{D}-\hat{L}) \hat{D}^{-1}(\hat{D}-\hat{U}),
$$

where $\hat{D}$ is a diagonal matrix; $\hat{L}$ and $\hat{U}$ are strictly lower triangular and upper triangular, respectively; the nonzero structure of $\hat{D}-\hat{L}-\hat{U}$ is the same as that of $A^{(b)}$; and the entries of $M$ are the same as the corresponding entries of $A^{(b)}$ wherever the latter are nonzero. We will examine the use of this factorization as a preconditioner for GMRES.

3. Analysis of separable problems and the ILU(0) factorization. We will be concerned with finite difference discretizations of (1.1). For example, on a uniform grid with mesh size $h$, let standard second order differences [11] be used for the second derivative terms. If centered differences are used for the first derivative terms, then after scaling by $h^{2}$, the values in the computational molecule are given by

$$
\begin{array}{ll}
a_{i j}=p\left(x_{i+1 / 2}, y_{j}\right)+p\left(x_{i-1 / 2}, y_{j}\right)+q\left(x_{i}, y_{j+1 / 2}\right)+q\left(x_{i}, y_{j-1 / 2}\right), \\
b_{i j}=-\left(q\left(x_{i}, y_{j-1 / 2}\right)+s\left(x_{i}, y_{j}\right) h / 2\right), \quad d_{i j}=-\left(p\left(x_{i+1 / 2}, y_{j}\right)-r\left(x_{i}, y_{j}\right) h / 2\right), \\
c_{i j}=-\left(p\left(x_{i-1 / 2}, y_{j}\right)+r\left(x_{i}, y_{j}\right) h / 2\right), \quad e_{i j}=-\left(q\left(x_{i}, y_{j+1 / 2}\right)-s\left(x_{i}, y_{j}\right) h / 2\right) .
\end{array}
$$

If upwind differencing is used for the first derivatives, then (for the case $r\left(x_{i}, y_{j}\right)>0$, $\left.s\left(x_{i}, y_{j}\right)>0\right)$ the values are

$$
\begin{gathered}
a_{i j}=p\left(x_{i+1 / 2}, y_{j}\right)+p\left(x_{i-1 / 2}, y_{j}\right)+q\left(x_{i}, y_{j+1 / 2}\right)+q\left(x_{i}, y_{j-1 / 2}\right) \\
+r\left(x_{i}, y_{j}\right) h+s\left(x_{i}, y_{j}\right) h, \\
b_{i j}=-\left(q\left(x_{i}, y_{j-1 / 2}\right)+s\left(x_{i}, y_{j}\right) h\right), \quad d_{i j}=-p\left(x_{i+1 / 2}, y_{j}\right), \\
c_{i j}=-\left(p\left(x_{i-1 / 2}, y_{j}\right)+r\left(x_{i}, y_{j}\right) h\right), \quad e_{i j}=-q\left(x_{i}, y_{j+1 / 2}\right) .
\end{gathered}
$$

If instead, $s\left(x_{i}, y_{j}\right)<0$, then $b_{i j}=-q\left(x_{i}, y_{j-1 / 2}\right), e_{i j}=-\left(q\left(x_{i}, y_{j+1 / 2}\right)-s\left(x_{i}, y_{j}\right) h\right)$, and $s\left(x_{i}, y_{j}\right) h$ is replaced by $-s\left(x_{i}, y_{j}\right) h$ in the expression for $a_{i j}$. The case $r\left(x_{i}, y_{j}\right)<$ 0 is handled in an analogous manner.

If $\Omega$ is a rectangular domain and the coefficients of (1.1a) satisfy

$$
p=p(x), \quad q=q(y), \quad r=r(x), \quad s=s(y),
$$

then the differential operator of (1.1) is separable [26]. In this case, the discrete coefficients of (1.3) satisfy

$$
\begin{aligned}
a_{i j} & =a_{i}^{(x)}+a_{j}^{(y)} \\
b_{i j} & =b_{j}, \quad c_{i j}=c_{i}, \quad d_{i j}=d_{i}, \quad e_{i j}=e_{j} .
\end{aligned}
$$


Our convergence analysis is based on symmetrizing the reduced matrix $A^{(b)}$ by a diagonal similarity transformation. The following result gives circumstances under which $A^{(b)}$ can be symmetrized when it comes from a separable operator. In the analysis, matrix entries are referenced using indices from the underlying reduced grid, as shown in Fig. 2.1. That is, every nonzero entry of the row of $A^{(b)}$ associated with the $(i, j)$ grid point is referenced using subscripts $i$ and $j$. For example, the entry corresponding to the point southwest of the center of the computational molecule (see Fig. 2.1) is denoted by

$$
-b_{j} c_{i}\left(\frac{1}{a_{i, j-1}}+\frac{1}{a_{i-1, j}}\right)
$$

where the numerator is expressed using the notation of (3.1).

THEOREM 1. If the operator of (1.1) is separable and $c_{i} d_{i-1}$ and $b_{j} e_{j-1}$ have the same sign for all $i$ and $j$, then the reduced matrix $A^{(b)}$ can be symmetrized with a real diagonal similarity transformation.

Proof. We seek a diagonal matrix $Q$ such that $Q^{-1} A^{(b)} Q$ is symmetric. Let $A^{(b)}$ be ordered by the natural one-line ordering, so that its rows and columns are grouped into $l$ blocks corresponding to $l$ individual lines. Let $Q$ be ordered the same way.

First consider the block diagonal $D$, which is a tridiagonal matrix. Any two successive rows of a block of $D$, corresponding to the $(i, j)$ and $(i-1, j+1)$ mesh points, contain the $2 \times 2$ sub-block

$$
\left(\begin{array}{cc}
* & -c_{i} e_{j}\left(\frac{1}{a_{i-1, j}}+\frac{1}{a_{i, j+1}}\right) \\
-b_{j+1} d_{i-1}\left(\frac{1}{a_{i-1, j}}+\frac{1}{a_{i, j+1}}\right) & *
\end{array}\right),
$$

where "*" denotes a diagonal entry. If $q_{i j}$ is known, then $q_{i-1, j+1}$ must be chosen so that

$$
q_{i-1, j+1}^{-1} b_{j+1} d_{i-1}\left(\frac{1}{a_{i-1, j}}+\frac{1}{a_{i, j+1}}\right) q_{i j}=q_{i j}^{-1} c_{i} e_{j}\left(\frac{1}{a_{i-1, j}}+\frac{1}{a_{i, j+1}}\right) q_{i-1, j+1} .
$$

Thus, within the blocks of $Q$, successive entries must satisfy

$$
q_{i-1, j+1}=\left(\frac{b_{j+1} d_{i-1}}{c_{i} e_{j}}\right)^{1 / 2} q_{i j}
$$

For symmetrizing $D$, the first entry of each block of $Q$ may be arbitrary.

To symmetrize the off-diagonal blocks of $A^{(b)}$, we require

$$
Q_{k}^{-1} A_{k, k-1}^{(b)} Q_{k-1}=\left(Q_{k-1}^{-1} A_{k-1, k}^{(b)} Q_{k}\right)^{T},
$$

where $k$ is a block (or line) index, $2 \leq k \leq l$. As in [7], there are three cases, corresponding to $2 \leq k<l / 2+1, k=l / 2+1$ ( $l$ even), and $l / 2+1<k$. In the case $2 \leq k \leq l / 2+1$, a careful specification of the entries of $Q$ and $A^{(b)}$ shows that (3.3) is equivalent to the following three scalar relations:

$$
\begin{gathered}
q_{i j}=\left(\frac{c_{i} c_{i-1}}{d_{i-1} d_{i-2}}\right)^{1 / 2} q_{i-2, j}, \\
q_{i-1, j+1}=\left(\frac{b_{j+1} c_{i-1}}{d_{i-2} e_{j}}\right)^{1 / 2} q_{i-2, j},
\end{gathered}
$$




$$
q_{i-2, j+2}=\left(\frac{b_{j+1} b_{j+2}}{e_{j} e_{j+1}}\right)^{1 / 2} q_{i-2, j}
$$

These relations specify three successive entries of $Q_{k}$ in terms of a single entry of $Q_{k-1}$ (where $k=(i+j-1) / 2$ ). Since the first entry of $Q_{k}$ is arbitrary, (3.4) can be used to define it. However, once this entry is defined, all subsequent entries are determined by (3.2). Thus, it is necessary to show that (3.4)-(3.6) are consistent with (3.2). But application of (3.2) and (3.4) in either order results in (3.5), showing that both (3.4) and (3.5) are consistent with (3.2). Similarly, (3.6) follows directly from (3.2) and (3.5).

The arguments for the cases $k=l / 2+1$ ( $l$ even) and $l / 2+1<k$ are essentially the same and we omit the details. A sufficient condition to guarantee that all the required square roots are well defined is that $c_{i} d_{i-1}$ and $b_{j} e_{j-1}$ have the same sign for all $i$ and $j$.

Finally, note that this analysis is not restricted to the natural one-line ordering. If $A^{(b)}$ is symmetrically permuted into some other order, giving the permuted matrix $\widetilde{A}^{(b)}$, then for an analogous permutation of $Q$ to $\widetilde{Q}, \widetilde{Q}^{-1} \widetilde{A}^{(b)} \widetilde{Q}$ is also symmetric.

Remark 1. For the centered difference discretization, necessary and sufficient conditions to ensure that all $c_{i} d_{i-1}$ and $b_{j} e_{j-1}$ have the same sign are that either

and

$$
\begin{aligned}
& \max _{i} {\left[\max \left(\left|\frac{r\left(x_{i}\right) h}{2 p\left(x_{i-1 / 2}\right)}\right|,\left|\frac{r\left(x_{i-1}\right) h}{2 p\left(x_{i-1 / 2}\right)}\right|\right)\right]<1 } \\
& \max _{j}\left[\max \left(\left|\frac{s\left(y_{j}\right) h}{2 q\left(y_{j-1 / 2}\right)}\right|,\left|\frac{s\left(y_{j-1}\right) h}{2 q\left(y_{j-1 / 2}\right)}\right|\right)\right]<1 ;
\end{aligned}
$$

or

and

$$
\begin{aligned}
\min _{i} & {\left[\min \left(\left|\frac{r\left(x_{i}\right) h}{2 p\left(x_{i-1 / 2}\right)}\right|\left|\frac{r\left(x_{i-1}\right) h}{2 p\left(x_{i-1 / 2}\right)}\right|\right)\right]>1 } \\
& \min _{j}\left[\min \left(\left|\frac{s\left(y_{j}\right) h}{2 q\left(y_{j-1 / 2}\right)}\right|,\left|\frac{s\left(y_{j-1}\right) h}{2 q\left(y_{j-1 / 2}\right)}\right|\right)\right]>1 .
\end{aligned}
$$

In contrast, the full system (1.2) can be symmetrized by a diagonal similarity transformation if and only if the conditions (3.7) hold. For upwind differences, it is always the case that $c_{i} d_{i-1}>0$ and $b_{j} e_{j-1}>0$ for all $i, j$.

Let $\hat{A}^{(b)}=Q^{-1} A^{(b)} Q$ denote the symmetrized reduced matrix, when it exists, for any of the strategies under consideration. Fig. 3.1 shows the resulting computational molecule. Let

$$
\hat{A}^{(b)}=\hat{D}-\hat{C}
$$

denote the block Jacobi splitting, where $\hat{D}=Q^{-1} D Q, \hat{C}=Q^{-1} C Q$. Note that $\hat{D}^{-1} \hat{C}=Q^{-1} D^{-1} C Q$, so that the eigenvalues of $D^{-1} C$ are the same as those of $\hat{D}^{-1} \hat{C}$, and in particular they are real. Let $\mathcal{L}_{\omega}=(D-\omega L)^{-1}[(1-\omega) D+\omega U]$ denote the block SOR iteration matrix. The following result is then a straightforward application of the analysis of the block SOR method [28].

COROLLARY 1. If $A^{(b)}$ is the reduced matrix derived from a separable operator, and $c_{i} d_{i-1}$ and $b_{j} e_{j-1}$ have the same sign for all $i$ and $j$, then $\rho\left(D^{-1} C\right)=\rho\left(\hat{D}^{-1} \hat{C}\right)$. If $\rho\left(D^{-1} C\right)<1$, then $\rho\left(\mathcal{L}_{\omega^{*}}\right)=\omega^{*}-1$, where $\omega^{*}=2 /\left(1+\sqrt{1+\left[\rho\left(D^{-1} C\right)\right]^{2}}\right)$ minimizes $\rho\left(\mathcal{L}_{\omega}\right)$. 


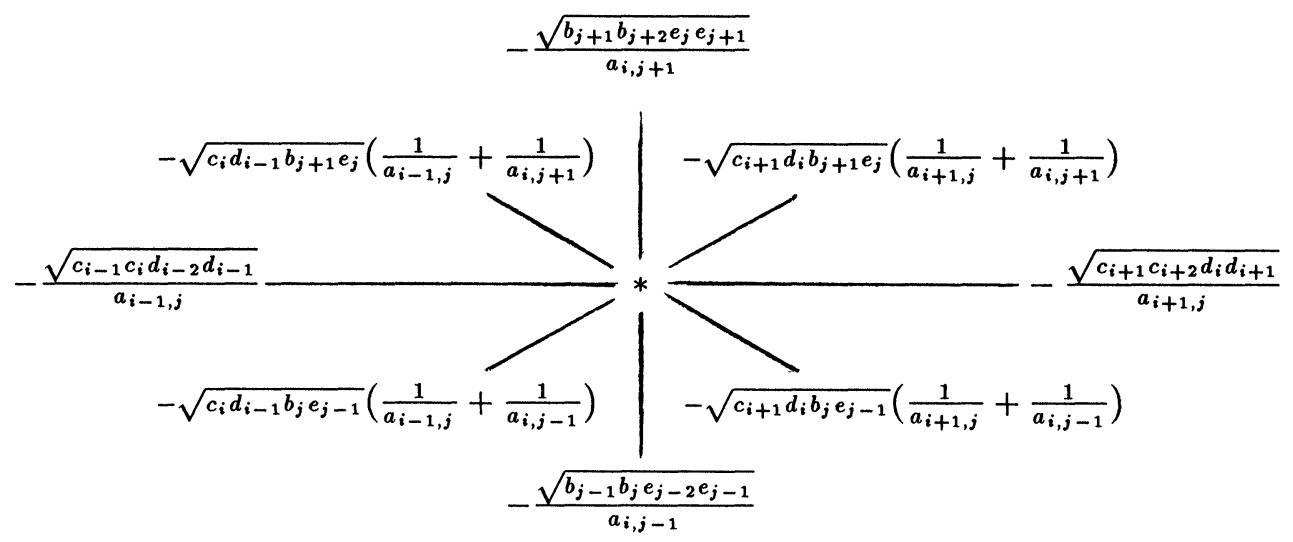

FIG. 3.1. The computational molecule for the symmetrized reduced system in the separable case.

Remark 2. It may be possible to establish the requirements of Corollary 1 a priori. Sufficient conditions to guarantee that $\rho\left(D^{-1} C\right)<1$ are that the original matrix $A$ be a diagonally dominant $M$-matrix, ${ }^{1}$ which is always the case for upwind differences, and is also true for centered differences for small enough $h$. (In addition, if $A$ is an $M$-matrix, then so is $A^{(b)}$ [10].) Even if Corollary 1 cannot be invoked from an a priori examination of matrix entries, it may still be useful as a guideline for practical computation. For example, for constant coefficient problems, empirical evidence and Fourier analysis suggest that $\rho\left(D^{-1} C\right)<1$ in cases where $c_{i} d_{i-1}$ and $b_{j} e_{j-1}$ are both negative but $A$ is not a diagonally dominant $M$-matrix. A good value for the SOR parameter could be computed from a dynamic estimation of $\rho\left(D^{-1} C\right)$, e.g., using the methods of $[14, \S 9]$. In addition, note that it is not necessary to compute $Q$ or $\hat{A}^{(b)}$ in order to apply this result, see [7].

The following result contains upper bounds on $\rho\left(D^{-1} C\right.$ ) (for both one-line and two-line splittings), for separable problems.

COROLlARY 2. Let $A^{(b)}$ come from a separable operator discretized on a uniform square grid of mesh width $h$, and assume that

$$
a_{i}^{(x)} \geq \alpha^{(x)}, \quad a_{j}^{(y)} \geq \alpha^{(y)}, \quad 0<c_{i+1} d_{i} \leq \xi, \quad 0<b_{j+1} e_{j} \leq \eta
$$

for all $i, j$. If $A^{(b)}=D-C$ is a one-line Jacobi splitting and

$$
\alpha^{(x)}+\alpha^{(y)} \geq \sqrt{2}(\sqrt{\xi}+\sqrt{\eta})
$$

then

$$
\rho\left(D^{-1} C\right) \leq \frac{2(\sqrt{\xi}+\sqrt{\eta})^{2}}{\left(\alpha^{(x)}+\alpha^{(y)}\right)^{2}-2(\sqrt{\xi}+\sqrt{\eta})^{2}+4 \sqrt{\xi \eta}(1-\cos \pi h)} .
$$

If $A^{(b)}=D-C$ is a two-line Jacobi splitting and

$$
\left(\alpha^{(x)}+\alpha^{(y)}\right)^{2} \geq 2(\sqrt{\xi}+\sqrt{\eta})^{2}+2 \xi
$$

\footnotetext{
1 A nonsingular matrix $X$ is an $M$-matrix if $X_{i j} \leq 0$ for $i \neq j$ and $X^{-1} \geq 0$.
} 
then

$$
\begin{aligned}
\rho\left(D^{-1} C\right) \leq & \frac{2 \eta \cos 2 \pi h+4 \sqrt{\xi \eta} \cos \pi h}{\left(\alpha^{(x)}+\alpha^{(y)}\right)^{2}-2(\sqrt{\xi}+\sqrt{\eta})^{2}-2 \xi+4 \sqrt{\xi \eta}(1-\cos \pi h)+4 \xi\left(1-\cos ^{2} \pi h\right)} \\
& +o\left(h^{2}\right) .
\end{aligned}
$$

Proof. Using Corollary 1, we have (for any ordering)

$$
\rho\left(D^{-1} C\right)=\rho\left(\hat{D}^{-1} \hat{C}\right) \leq\left\|\hat{D}^{-1}\right\|_{2}\|\hat{C}\|_{2}=\rho(\hat{D}) \rho(\hat{C}) .
$$

Consider the one-line orderings. By (3.8), all nonzero off-diagonal entries of $\hat{D}$ are bounded below by $-2 \sqrt{\xi \eta} /\left(\alpha^{(x)}+\alpha^{(y)}\right)$, and all diagonal entries of $\hat{D}$ are bounded below by

$$
\alpha^{(x)}+\alpha^{(y)}-2 \xi /\left(\alpha^{(x)}+\alpha^{(y)}\right)-2 \eta /\left(\alpha^{(x)}+\alpha^{(y)}\right) .
$$

Thus, $\hat{D} \geq \widetilde{D}$, where each block of $\widetilde{D}$ is a constant coefficient tridiagonal matrix

$$
\operatorname{tri}\left[-\frac{2 \sqrt{\xi \eta}}{\alpha^{(x)}+\alpha^{(y)}}, \alpha^{(x)}+\alpha^{(y)}-\frac{2 \xi}{\alpha^{(x)}+\alpha^{(y)}}-\frac{2 \eta}{\alpha^{(x)}+\alpha^{(y)}},-\frac{2 \sqrt{\xi \eta}}{\alpha^{(x)}+\alpha^{(y)}}\right] \text {. }
$$

The size of this block depends on the line from which it is derived. Assumption (3.9) implies that each block (3.13) and, therefore, each corresponding block of $\hat{D}$, is an irreducibly diagonally dominant $M$-matrix. Hence, the Perron-Frobenius theory implies $\rho\left(\hat{D}^{-1}\right) \leq \rho\left(\widetilde{D}^{-1}\right)$. Similarly, by $(3.8), 0 \leq \hat{C} \leq \widetilde{C}$, where $\widetilde{C}$ is a matrix with the same nonzero structure as that of $\hat{C}$ in which all occurences of $c_{i} d_{i-1}, b_{j} e_{j-1}$, and $a_{i j}$ are replaced by $\xi, \eta$, and $\alpha^{(x)}+\alpha^{(y)}$, respectively. Consequently, $\rho(\hat{C}) \leq \rho(\widetilde{C})$, and we have

$$
\rho\left(\hat{D}^{-1}\right) \rho(\hat{C}) \leq \rho\left(\widetilde{D}^{-1}\right) \rho(\widetilde{C}),
$$

where the right side of the inequality contains constant coefficient matrices. The bound (3.10) is determined from the maximum eigenvalue of $\widetilde{D}^{-1}$ and use of Gerschgorin's theorem for $\widetilde{C}$. (See [7, Thm. 4])

For the two-line ordering, the blocks of $D$ and $\hat{D}$ are pentadiagonal matrices, and $\hat{D} \geq \widetilde{D}$, where each block of $\widetilde{D}$ is a constant coefficient pentadiagonal matrix,

$$
\begin{gathered}
\text { penta }\left[-\frac{\xi}{\alpha^{(x)}+\alpha^{(y)}},-\frac{2 \sqrt{\xi \eta}}{\alpha^{(x)}+\alpha^{(y)}}, \alpha^{(x)}+\alpha^{(y)}-\frac{2 \xi}{\alpha^{(x)}+\alpha^{(y)}}-\frac{2 \eta}{\alpha^{(x)}+\alpha^{(y)}},\right. \\
\left.-\frac{2 \sqrt{\xi \eta}}{\alpha^{(x)}+\alpha^{(y)}},-\frac{\xi}{\alpha^{(x)}+\alpha^{(y)}}\right]
\end{gathered}
$$

which is assumed in (3.11) to be diagonally dominant. In addition, exactly as above, $0 \leq \hat{C} \leq \widetilde{C}$, where $\widetilde{C}$ has the same nonzero structure as $\hat{C}$. The bound (3.12) then follows from [8, Thm. 5].

We will examine the use of this result in $\S 5$.

Remark 3. In the interest of brevity, we have limited our attention to the natural and red-black variants of the one-line orderings. Other variants, called "torus" oneline orderings, collect some individual lines together into sets of equal sizes; this is 
useful for parallel and vector computations. (See [8], [16].) All of the analysis of this section also applies to the torus orderings.

We now turn our attention to incomplete LU (ILU) factorizations. Let $B$ be an $M$-matrix of order $N$, and let $\mathcal{N} \subseteq\{(i, j) \mid 1 \leq i, j \leq N\}$ be an index set containing all diagonal indices $(i, i)$. It is shown in [17] that there is a unique ILU factorization $L U$ such that $L$ is unit lower triangular, $U$ is upper triangular, $l_{i j}=0$ and $u_{i j}=0$ for $(i, j) \notin \mathcal{N}$, and $[L U-B]_{i j}=0$ for $(i, j) \in \mathcal{N}$. The ILU(0) factorization of (2.4) is a particular example. The following result of Beauwens ([2, Thm. 4.4]) can be used to compare the ILU(0) splitting to the block Jacobi splitting.

THEOREM 2. Let $B$ be a nonsingular $M$-matrix, and let

$$
B=M_{1}-R_{1}=M_{2}-R_{2},
$$

where $M_{1}=L_{1} U_{1}$ and $M_{2}=L_{2} U_{2}$ are incomplete factorizations of $B$ such that the set of matrix indices for which $L_{1}+U_{1}$ is permitted to be nonzero is contained in the set of indices for which $L_{2}+U_{2}$ is permitted to be nonzero. Then

$$
\rho\left(M_{2}^{-1} R_{2}\right) \leq \rho\left(M_{1}^{-1} R_{1}\right) .
$$

The analysis in [2] actually applies to a more general class of factorizations than the standard ILU factorization. Theorem 2 can be proved using the result of Woz̀nicki [27], that if (3.15) represents two regular splittings of a matrix $B$ for which $B^{-1} \geq 0$, then

$$
M_{2}^{-1} \geq M_{1}^{-1}
$$

implies the conclusion (3.16). It is straightforward to establish (3.17) for ILU factorizations.

COROLlaRY 3. Suppose $A^{(b)}$ is an $M$-matrix, ordered using any of the orderings under consideration. Let $A^{(b)}=M-R$ where $M$ is the $\mathrm{ILU}(0)$ factorization of $A^{(b)}$, and let $A^{(b)}=D-C$ denote the block Jacobi splitting. Then $\rho\left(M^{-1} R\right) \leq \rho\left(D^{-1} C\right)$.

Proof. The index set of nonzeros of the block diagonal $D$ is a proper subset of the nonzero index set for the ILU(0) factorization. The result then follows from Theorem 2, where (the factorization of) $D$ is viewed as an incomplete factorization of $A^{(b)}$.

Thus, we expect convergence of a stationary method based on the ILU(0) splitting to be at least as fast as that for the block Jacobi method, for any ordering. (The work per step for the Jacobi method will be smaller, though.) In particular, as observed in [7], [8], convergence should be faster for mildly nonsymmetric problems arising from nonselfadjoint operators than for symmetric ones derived from selfadjoint operators. Combining the ILU(0) factorization with an acceleration scheme such as GMRES (i.e., using $M$ as a preconditioner) should further improve convergence. Numerical experiments with the ILU $(0)$ preconditioner that support this statement are presented in the following sections.

4. Experimental results: Constant coefficient problems. In this section, we examine the numerical performance of the block Gauss-Seidel and SOR stationary methods, and GMRES(5) with the ILU(0) preconditioner, for solving the constant coefficient model problem

$$
-\Delta u+\sigma u_{x}+\tau u_{y}=0
$$



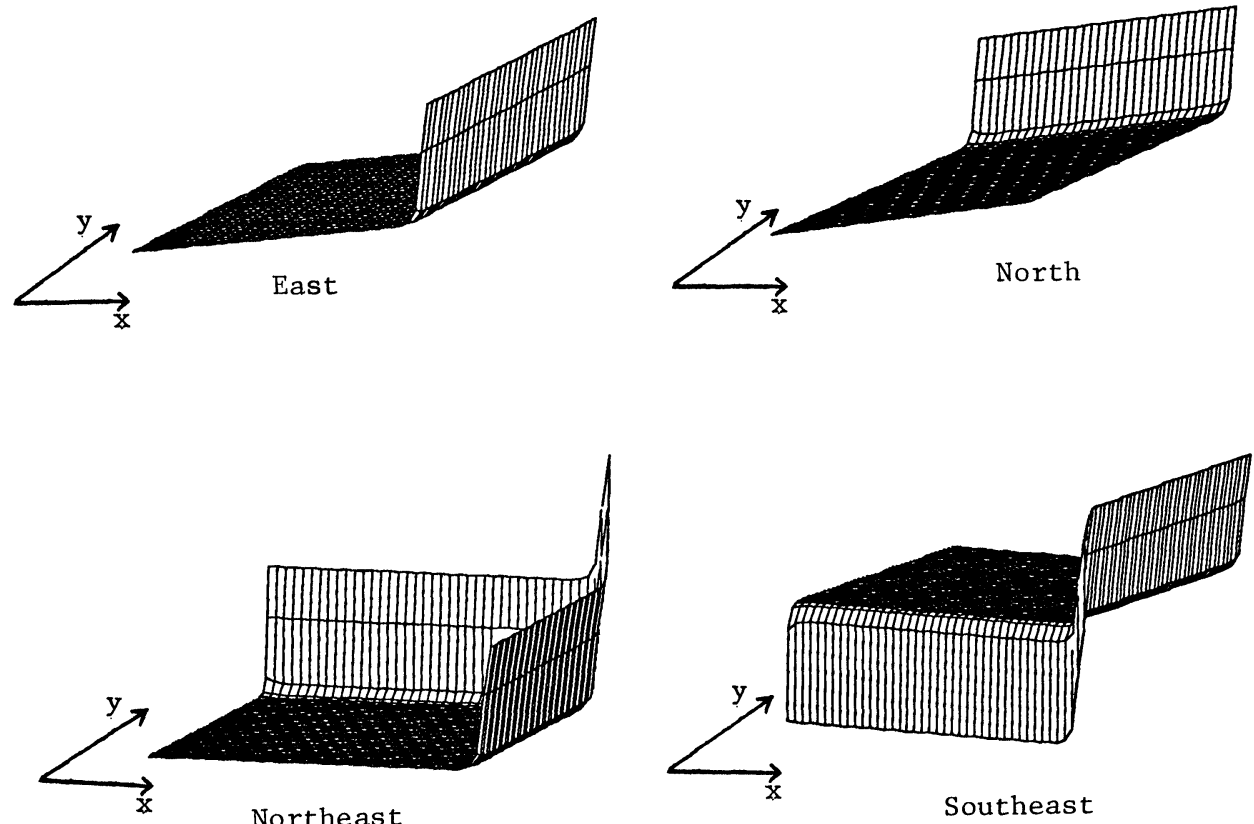

FIG. 4.1. Plots of the constant coefficient solution for four different directions of flow.

on $\Omega=(0,1) \times(0,1)$. Dirichlet boundary conditions on $\partial \Omega$ are determined from the exact solution

$$
u(x, y)=\frac{e^{\sigma x}-1}{e^{\sigma}-1}+\frac{e^{\tau y}-1}{e^{\tau}-1}
$$

on $\bar{\Omega}$. The vector $(\sigma, \tau)$ represents a velocity field with the signs of $\sigma$ or $\tau$ determining the direction of flow. We consider eight types of velocity fields, corresponding to eight flow directions in the $(x, y)$-plane:

$$
\begin{array}{llll}
\text { East (E): } & \sigma>0, \tau=0, & \text { Northeast (NE): } & \sigma=\tau>0, \\
\text { West (W): } & \sigma<0, \tau=0, & \text { Southeast (SE): } & \sigma=-\tau>0, \\
\text { North (N): } & \sigma=0, \tau>0, & \text { Northwest (NW): } & \sigma=-\tau<0, \\
\text { South (S): } & \sigma=0, \tau<0, & \text { Southwest (SW): } & \sigma=\tau<0 .
\end{array}
$$

(For $\sigma=0$ or $\tau=0,(4.2)$ is defined using the limit, i.e., $\lim _{\sigma \rightarrow 0}\left(e^{\sigma x}-1\right) /\left(e^{\sigma}-1\right)=x$.) In addition, the solution (4.2) has a boundary layer at any outflow boundary, i.e., near $x=1$ for positive $\sigma$ and $x=0$ for negative $\sigma$, and similarly for $y$ and $\tau$. Plots of the solution for four such $(\sigma, \tau)$ combinations, corresponding to flows in the east, north, northeast, and southeast directions, are shown in Fig. 4.1. Our concern is to determine the effects of direction and magnitude of flow, ordering of unknowns, discretization scheme, and use of local mesh refinement on the performance of reduced system iterative methods.

Details of the numerical experiments are as follows. The experiments were performed on a VAX-8600 in double precision Fortran. Reported iteration counts are averages over three initial guesses consisting of vectors of random numbers in $[-1,1]$. The stopping criterion for all methods was $\left\|r_{i}\right\|_{2} /\left\|r_{0}\right\|_{2} \leq 10^{-6}$. A maximum of 150 iterations was permitted; an asterisk " $*$ " in any table entry below indicates that for at least one initial guess, the stopping criterion was not met after 150 steps. (We 
remark that when the block stationary methods failed to meet the stopping criterion, they never "stagnated," i.e., they appeared to be converging.) For red-black SOR, the first iteration was performed with $\omega=1$, as in [24]. Preconditioned GMRES was performed with right-oriented preconditioning, i.e., GMRES was applied to the preconditioned problem $A^{(b)} M^{-1} \hat{u}^{(b)}=g^{(b)}$, where $M$ is the preconditioning matrix and $u^{(b)}=M^{-1} \hat{u}^{(b)}$. The construction of the reduced matrices and the experiments with GMRES were performed with PCGPAK [21].

TABLE 4.1

Average iteration counts for the natural one-line ordering, for eight flow directions.

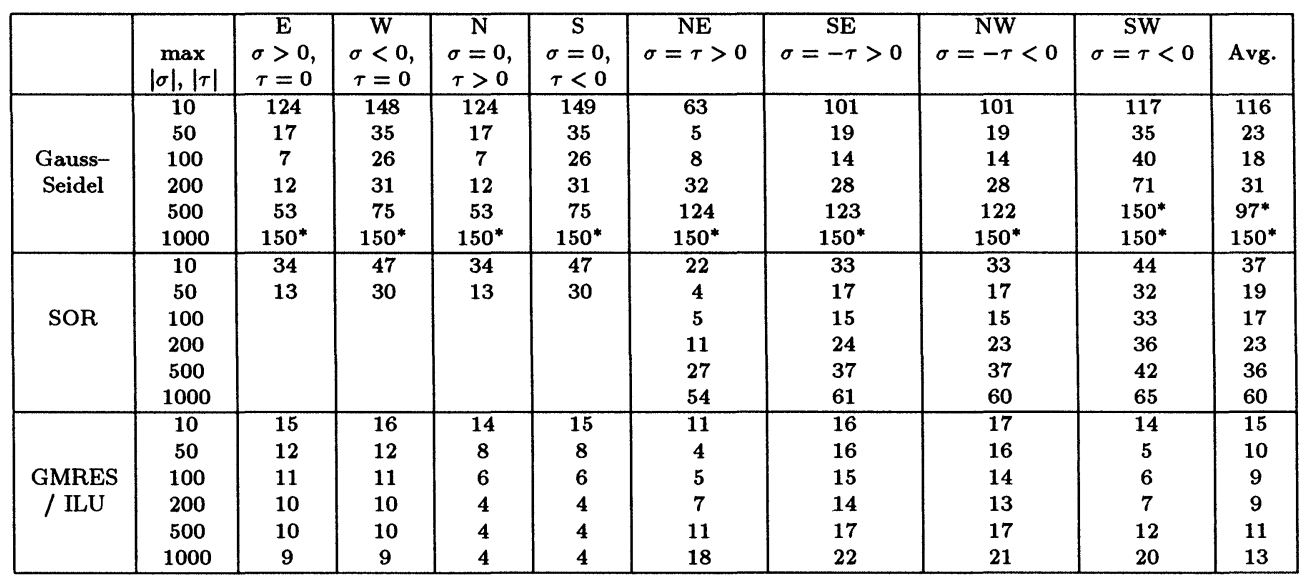

TABLE 4.2

Average iteration counts for the red-black one-line ordering, for eight flow directions.

\begin{tabular}{|c|c|c|c|c|c|c|c|c|c|c|}
\hline & $\begin{array}{c}\max \\
|\sigma|,|\tau|\end{array}$ & $\begin{array}{c}\mathrm{E} \\
\sigma>0 \\
\tau=0\end{array}$ & $\begin{array}{c}\text { W } \\
\sigma<0 \\
\tau=0\end{array}$ & $\begin{array}{c}N \\
\sigma=0 \\
\tau>0\end{array}$ & $\begin{array}{c}\mathrm{S} \\
\sigma=0 \\
\tau<0\end{array}$ & $\begin{array}{c}\mathrm{NE} \\
\sigma=\tau>0\end{array}$ & $\sigma=-\tau=0$ & $\begin{array}{c}\text { NW } \\
\sigma=-\tau<0\end{array}$ & $\begin{array}{c}\text { SW } \\
\sigma=\tau<0\end{array}$ & Avg. \\
\hline \multirow{6}{*}{$\begin{array}{l}\text { Gauss- } \\
\text { Seidel }\end{array}$} & 10 & 132 & 144 & 133 & 144 & 82 & 103 & 103 & 108 & 119 \\
\hline & 50 & 23 & 24 & 23 & 24 & 19 & 18 & 18 & 21 & 23 \\
\hline & 100 & 13 & 14 & 13 & 14 & 22 & 11 & 11 & 26 & 15 \\
\hline & 200 & 20 & 21 & 20 & 21 & 49 & 27 & 27 & 57 & 30 \\
\hline & 500 & 63 & 69 & 63 & 69 & $140^{*}$ & 128 & 128 & $150^{*}$ & $102^{*}$ \\
\hline & 1000 & $150^{*}$ & $150^{*}$ & $150^{*}$ & $150^{*}$ & $150^{*}$ & $150^{*}$ & $150^{*}$ & $150^{*}$ & $150^{*}$ \\
\hline \multirow{6}{*}{ SOR } & 10 & 33 & 34 & 33 & 34 & 27 & 29 & 30 & 28 & 31 \\
\hline & 50 & 23 & 24 & 23 & 24 & 19 & 18 & 18 & 21 & 21 \\
\hline & 100 & & & & & 18 & 14 & 14 & 19 & 16 \\
\hline & 200 & & & & & 21 & 23 & 22 & 22 & 22 \\
\hline & 500 & & & & & 31 & 35 & 34 & 33 & 33 \\
\hline & 1000 & & & & & 57 & 58 & 57 & 57 & 57 \\
\hline \multirow{6}{*}{$\begin{array}{l}\text { GMRES } \\
\text { / ILU }\end{array}$} & 10 & 24 & 28 & 25 & 30 & 27 & 29 & 27 & 32 & 28 \\
\hline & 50 & 29 & 35 & 26 & 35 & 37 & 22 & 20 & 51 & 32 \\
\hline & 100 & 28 & 33 & 27 & 35 & 38 & 16 & 16 & 53 & 31 \\
\hline & 200 & 28 & 34 & 28 & 34 & 37 & 14 & 14 & 53 & 30 \\
\hline & 500 & 31 & 34 & 31 & 33 & 35 & 27 & 26 & 49 & 33 \\
\hline & 1000 & 39 & 42 & 39 & 43 & 46 & 52 & 52 & 53 & 46 \\
\hline
\end{tabular}


TABLE 4.3

Average iteration counts for the natural two-line ordering, for eight flow directions.

\begin{tabular}{|c|c|c|c|c|c|c|c|c|c|c|}
\hline & $\underset{|\sigma|,|\tau|}{\max }$ & $\begin{array}{c}\mathrm{E} \\
\sigma>0 \\
\tau=0\end{array}$ & $\begin{array}{c}W \\
\sigma<0 \\
\tau=0\end{array}$ & $\begin{array}{c}\mathrm{N} \\
\sigma=0 \\
\tau>0\end{array}$ & $\begin{array}{c}\mathrm{S} \\
\sigma=0 \\
\tau<0\end{array}$ & $\begin{array}{c}\mathrm{NE} \\
\sigma=\tau>0\end{array}$ & $\sigma=-\tau>0$ & $\begin{array}{c}\text { NW } \\
\sigma=-\tau<0\end{array}$ & $\begin{array}{c}\text { SW } \\
\sigma=\tau<0\end{array}$ & Avg. \\
\hline \multirow{6}{*}{$\begin{array}{l}\text { Gauss- } \\
\text { Seidel }\end{array}$} & 10 & 101 & 109 & 92 & 115 & 50 & 84 & 72 & 87 & 89 \\
\hline & 50 & 22 & 23 & 9 & 25 & 7 & 22 & 8 & 23 & 18 \\
\hline & 100 & 13 & 13 & 8 & 23 & 6 & 21 & 7 & 21 & 14 \\
\hline & 200 & 9 & 9 & 15 & 31 & 13 & 28 & 14 & 28 & 19 \\
\hline & 500 & 6 & 6 & 52 & 64 & 47 & 63 & 53 & 64 & 44 \\
\hline & 1000 & 5 & 5 & $150^{*}$ & $150^{*}$ & 143 & $150^{*}$ & $148^{*}$ & $150^{*}$ & $117^{*}$ \\
\hline \multirow{6}{*}{ SOR } & 10 & 30 & 31 & 22 & 33 & 25 & 37 & 26 & 38 & 30 \\
\hline & 50 & 19 & 20 & 6 & 20 & 6 & 21 & 8 & 22 & 15 \\
\hline & 100 & & & & & 9 & 25 & 11 & 25 & 17 \\
\hline & 200 & & & & & 16 & 29 & 17 & 29 & 23 \\
\hline & 500 & & & & & 31 & 41 & 31 & 41 & 36 \\
\hline & 1000 & & & & & 56 & 64 & 56 & 65 & 60 \\
\hline \multirow{6}{*}{$\begin{array}{c}\text { GMRES } \\
\text { / ILU }\end{array}$} & 10 & 17 & 16 & 17 & 17 & 12 & 19 & 18 & 18 & 17 \\
\hline & 50 & 12 & 13 & 12 & 13 & 5 & 27 & 25 & 5 & 10 \\
\hline & 100 & 10 & 10 & 10 & 11 & 5 & 30 & 30 & 5 & 14 \\
\hline & 200 & 8 & 8 & 8 & 9 & 10 & 33 & 30 & 10 & 14 \\
\hline & 500 & 7 & 7 & 8 & 8 & 22 & 43 & 41 & 22 & 20 \\
\hline & 1000 & 6 & 6 & 8 & 8 & 45 & 49 & 49 & 48 & 28 \\
\hline
\end{tabular}

TABLE 4.4

Average iteration counts for the red-black two-line ordering, for eight flow directions.

\begin{tabular}{|c|c|c|c|c|c|c|c|c|c|c|}
\hline & $\underset{|\sigma|,|\tau|}{\max }$ & $\begin{array}{c}\mathrm{E} \\
\sigma>0 \\
\tau=0\end{array}$ & $\begin{array}{c}\mathrm{W} \\
\sigma<0 \\
\tau=0\end{array}$ & $\begin{array}{c}N \\
\sigma=0 \\
\tau>0\end{array}$ & $\begin{array}{c}\mathrm{S} \\
\sigma=0 \\
\tau<0\end{array}$ & $\begin{array}{c}\mathrm{NE} \\
\sigma=\tau>0\end{array}$ & $\begin{array}{c}\mathrm{SE} \\
\sigma=-\tau>0\end{array}$ & $\begin{array}{c}\text { NW } \\
\sigma=-\tau<0\end{array}$ & $\begin{array}{c}\text { SW } \\
\sigma=\tau<0\end{array}$ & Avg. \\
\hline \multirow{6}{*}{$\begin{array}{l}\text { Gauss- } \\
\text { Seidel }\end{array}$} & 10 & 100 & 110 & 100 & 109 & 60 & 78 & 78 & 82 & 90 \\
\hline & 50 & 19 & 20 & 17 & 18 & 14 & 15 & 15 & 16 & 17 \\
\hline & 100 & 10 & 11 & 15 & 16 & 13 & 14 & 13 & 14 & 13 \\
\hline & 200 & 8 & 8 & 22 & 24 & 20 & 21 & 21 & 21 & 18 \\
\hline & 500 & 6 & 6 & 56 & 58 & 54 & 56 & 59 & 57 & 44 \\
\hline & 1000 & 5 & 5 & $150^{*}$ & $150^{*}$ & 146 & $150^{*}$ & $150^{*}$ & $149^{*}$ & $113^{*}$ \\
\hline \multirow{6}{*}{ SOR } & 10 & 24 & 26 & 24 & 25 & 28 & 29 & 29 & 29 & 26 \\
\hline & 50 & 15 & 16 & 13 & 14 & 13 & 14 & 14 & 15 & 14 \\
\hline & 100 & & & & & 17 & 17 & 17 & 17 & 17 \\
\hline & 200 & & & & & 21 & 23 & 22 & 23 & 22 \\
\hline & 500 & & & & & 34 & 35 & 35 & 35 & 35 \\
\hline & 1000 & & & & & 58 & 58 & 58 & 58 & 58 \\
\hline \multirow{6}{*}{$\begin{array}{l}\text { GMRES } \\
\text { / ILU }\end{array}$} & 10 & 20 & 21 & 20 & 23 & 16 & 23 & 23 & 25 & 21 \\
\hline & 50 & 12 & 13 & 25 & 31 & 15 & 23 & 24 & 25 & 21 \\
\hline & 100 & 8 & 9 & 26 & 30 & 16 & 22 & 24 & 25 & 20 \\
\hline & 200 & 6 & 7 & 26 & 30 & 17 & 23 & 23 & 28 & 20 \\
\hline & 500 & 8 & 9 & 34 & 29 & 24 & 30 & 28 & 31 & 24 \\
\hline & 1000 & 7 & 8 & 40 & 43 & 36 & 42 & 41 & 45 & 33 \\
\hline
\end{tabular}

The orientation of line orderings was as in $\S 2$. That is, for the one-line orderings, lines were oriented in the NW-SE direction, and the natural ordering arranged the lines starting from the SW corner; and for the two-line orderings, line pairs were grouped by horizontal lines and the natural listing is from bottom (south) to top (north). Note that the lines associated with ordering strategies have a relationship with the direction of flow (see also [4]). For example, for the natural one-line ordering, when the flow direction is NE, the lines are perpendicular to the direction of flow, and the Gauss-Seidel and SOR sweeps follow the flow. When the flow direction is $\mathrm{SW}$, the lines are perpendicular to flow, but the sweeps are in the opposite direction 
of the flow. On the other hand, the sweeps for the red-black orderings do not have a clear relationship to the direction of flow (although the line orientations still do). The ILU(0) preconditioning entails lower and upper triangular solves, so that, for the natural line orderings, the preconditioning operation can be thought of as a pair of bidirectional sweeps.

Tables 4.1-4.4 contain results for centered difference discretizations on a uniform mesh of width $h=1 / 32$. For this class of problems, the analysis of $\S 3$ is applicable when $|\sigma h / 2|$ and $|\tau h / 2|$ are both less than one, i.e., when $\sigma$ or $\tau$ are 10 or 50 in the problems considered. In these cases, Corollary 1 is used to choose the SOR parameter $\omega$, where $\rho\left(D^{-1} C\right)$ is approximated using the bounds (3.10) and 3.12; here

$$
a_{i}^{(x)}=\alpha^{(x)}=a_{j}^{(y)}=\alpha^{(y)}=2, \quad \xi=1-(\sigma h / 2)^{2}, \quad \eta=1-(\tau h / 2)^{2} .
$$

For the one-line orderings, when both $|\sigma h / 2|$ and $|\tau h / 2|$ are greater than one, the Fourier analysis of [7] can be used to estimate $\rho\left(D^{-1} C\right)$, from which good values of $\omega$ are also obtained (i.e., using the formula for $\omega^{*}$ in Corollary 1 ). These values were also used for the two-line orderings when $|\sigma h / 2|>1$ and $|\tau h / 2|>1$, although there is no theoretical justification for this. We did not examine SOR when one of $|\sigma h / 2|,|\tau h / 2|$ is greater than one and the other is less than one. Table 4.5 shows the choices of $\omega$ used for Tables 4.1-4.4. Note that the analysis of $\S 3$ and [7], [8], does not distinguish between natural and red-black orderings, or between problems where the magnitudes of $\sigma($ or $\tau$ ) are the same but the signs differ.

TABLE 4.5

Values of SOR parameters used for Tables 4.1-4.4.

\begin{tabular}{|c|c|c|c|c|c|}
\hline & \multicolumn{2}{|c|}{ One-line orderings } & \multicolumn{3}{c|}{ Two-line orderings } \\
\hline $\max$ & $\mathrm{E} / \mathrm{W} / \mathrm{N} / \mathrm{S}$ & $\mathrm{NE} / \mathrm{SE} / \mathrm{NW} / \mathrm{SW}$ & $\mathrm{E} / \mathrm{W}$ & $\mathrm{N} / \mathrm{S}$ & NE/SE/NW/SW \\
\hline 10 & 1.63 & 1.52 & 1.52 & 1.52 & 1.44 \\
50 & 1.07 & 1.02 & 1.06 & 1.04 & 1.01 \\
100 & & 1.05 & & & 1.05 \\
200 & & 1.27 & & & 1.27 \\
500 & & 1.60 & & & 1.60 \\
1000 & & 1.77 & & & 1.77 \\
\hline
\end{tabular}

We make the following observations on the data of Tables 4.1-4.4:

1. For the stationary methods (Gauss-Seidel and SOR), performance depends on the relationship between flow direction and sweep direction, but the effects vary depending on the magnitudes of the velocity vectors. For example, for the natural one-line orderings, when the convection terms are small or moderate in size, the best performance of the Gauss-Seidel and SOR methods occurs when the sweeps follow the flow (i.e., when the flow direction is $\mathrm{NE}$ ). When the convection terms dominate, the stationary methods perform better when the flow direction forms a nonzero acute angle with the sweep direction (flow is $\mathrm{N}$ or $\mathrm{E}$ ), than when the sweeps follow the flow. For the natural two-line ordering, performance for moderate sized convection terms is best when the flow direction forms an acute angle with the sweep direction (i.e., when flow is $\mathrm{N}, \mathrm{NE}$, or NW); for convection-dominated systems, performance is best when the sweep is perpendicular to the flow. It is always the case that sweeping in the opposite direction of the flow is a bad choice.

2. Performance of stationary methods for the red-black orderings is much less sensitive to flow directions. In particular, the average iteration counts (over the eight flow directions) are essentially the same for the natural and red-black orderings. 
This is significant on parallel architectures, where the red-black orderings can be implemented more efficiently [8]. The minimum iteration counts are typically lower for the natural orderings than for the red-black orderings.

3. Somewhat different conclusions apply for GMRES/ILU. There is no clear correlation between direction of flow and performance, except that for convectiondominated problems, performance for both natural orderings degrades when the directions of flow are not parallel to one of the grid coordinates. We have no simple explanation for this. The average iteration counts for GMRES/ILU are typically higher for the red-black orderings than for the natural orderings. Similar results have been obtained for symmetric problems, with point red-black and natural orderings, e.g., in [1].

4. One step of the block SOR method is approximately as expensive as one matrix-vector product and one scalar-vector product [8]. Thus, its cost per step is approximately $10 N_{b}$ multiply-adds, where $N_{b}$ is the order of $A^{(b)}$. One step of GMRES(5) with ILU(0) preconditioning entails a preconditioning solve, a matrixvector product, and approximately $8 N_{b}$ vector operations [23], for a total cost of $26 N_{b}$ multiply-adds. That is, one GMRES/ILU step is about 2.5 times as expensive as one SOR step. Consequently, the performances of the stationary methods and GMRES/ILU are comparable for problems with small and moderate-sized convection terms (where for problems with small convection terms, it is necessary to use a good SOR parameter to achieve good performance). GMRES/ILU is somewhat more effective for convection-dominated systems, especially when there is no simple way of choosing a relaxation parameter. GMRES(5) requires $7 N_{b}$ storage locations [23], plus approximately $9 N_{b}$ for the factors of $M$. SOR requires essentially one vector of storage for the solution iterates $\left\{u_{b}^{(k)}\right\}$, plus storage for the factors of the block diagonal $D$. If no pivoting is required, these factors could overwrite the analogous locations of $A^{(b)}$.

TABLE 4.6

Average iteration counts for the block Gauss-Seidel method, upwind differences.

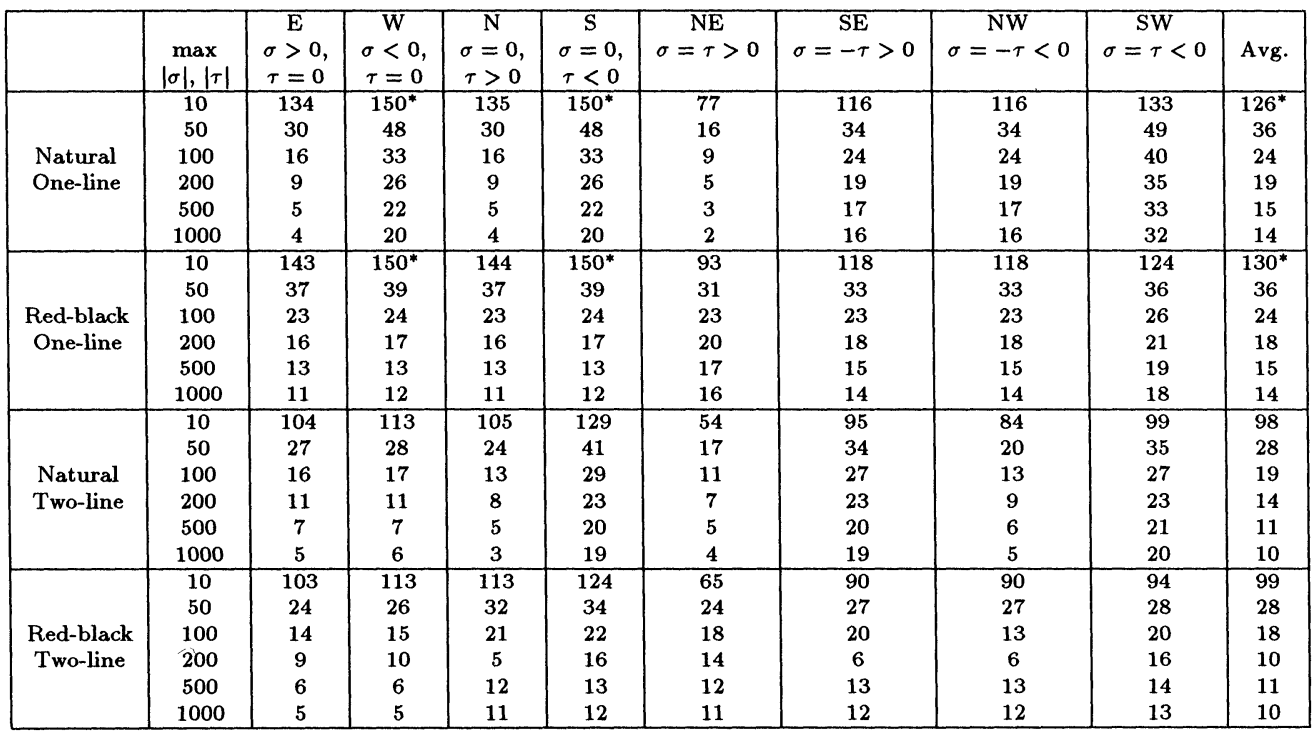


Table 4.6 shows the performance of the block Gauss-Seidel method for solving the same set of problems using the upwind difference scheme for the first derivative terms. The main difference from the results for centered differences is that performance improves as $\sigma$ or $\tau$ increases. This is because $A^{(b)}$ (as well as $A$ ) becomes more diagonally dominant in these cases. In addition, for the natural one-line ordering, performance is consistently best when the flow is in the same direction as the sweep (NE), and good performance is achieved when the sweep and flow directions make an acute angle. Similar observations apply for the natural two-line ordering, except that sweeping in the direction of flow $(\mathrm{N})$ is not best when the convection terms are small. As above, the red-black orderings tend to be less sensitive than the natural orderings to flow directions.

The results above do not address the issue of accuracy of the discrete solution. If $|\sigma h / 2|$ or $|\tau h / 2|$ is greater than one and boundary layers are present in the continuous solution, then the discrete solution tends to be inaccurate near the boundary layers, and it is oscillatory when centered differences are used [22]. If the boundary layer can be located, then one possible remedy is to use local mesh refinement. For the solution (4.2), for nonzero $\sigma$ or $\tau$, there are boundary layers of width $O(1 / \sigma)$ (or $O(1 / \tau)$ ) near the outflow boundary. We consider one local refinement strategy, which we describe in terms of the "horizontal" parameters $x$ and $\sigma$. In the interval of width $2 / \sqrt{\sigma}$ containing the boundary layer (at either $x=0$ or $x=1$ ), we use a mesh of size $\tilde{h}$ such that $|\sigma \tilde{h} / 2|=.75$; away from that interval, we use $h=1 / 32 .^{2}$ It was shown in [6] that this strategy does a good job of resolving the boundary layer with the addition

TABLE 4.7

Average iteration counts for the natural one-line ordering, centered differences and local mesh refinement.

\begin{tabular}{|c|c|c|c|c|c|c|c|c|c|c|}
\hline & \begin{tabular}{|}
$\max$ \\
$|\sigma|,|\tau|$
\end{tabular} & $\begin{array}{c}\mathrm{E} \\
\sigma>0 \\
\tau=0\end{array}$ & $\begin{array}{c}\text { W } \\
\sigma<0 \\
\tau=0\end{array}$ & $\begin{array}{c}\mathrm{N} \\
\sigma=0, \\
\tau>0\end{array}$ & $\begin{array}{c}\mathrm{S} \\
\sigma=0, \\
\tau<0\end{array}$ & $\begin{array}{c}\mathrm{NE} \\
\sigma=\tau>0\end{array}$ & $\begin{array}{c}\mathrm{SE} \\
\sigma=-\tau>0\end{array}$ & $\begin{array}{c}\text { NW } \\
\sigma=-\tau<0\end{array}$ & $\begin{array}{c}\text { SW } \\
\sigma=\tau<0\end{array}$ & Avg. \\
\hline \multirow{4}{*}{$\begin{array}{c}\text { Gauss- } \\
\text { Seidel }\end{array}$} & 100 & 7 & 31 & 7 & 31 & 8 & 17 & 17 & 47 & 21 \\
\hline & 200 & 12 & 37 & 12 & 37 & 32 & 28 & 28 & 80 & 33 \\
\hline & 500 & 46 & 73 & 46 & 73 & 124 & 111 & 109 & $150^{*}$ & $91^{*}$ \\
\hline & 1000 & 134 & $150^{*}$ & 132 & $150^{*}$ & & & & & \\
\hline \multirow{4}{*}{$\begin{array}{l}\text { GMRES } \\
\text { / ILU }\end{array}$} & 100 & 12 & 12 & 6 & 6 & 6 & 17 & 17 & 6 & 10 \\
\hline & 200 & 10 & 10 & 4 & 4 & 8 & 18 & 17 & 8 & 10 \\
\hline & 500 & 10 & 10 & 4 & 3 & 12 & 18 & 21 & 11 & 11 \\
\hline & 1000 & 9 & 9 & 4 & 2 & 18 & 23 & 24 & 14 & 13 \\
\hline
\end{tabular}

\footnotetext{
${ }^{2}$ Grid points are distributed from left to right within each of these subintervals, so that the rightmost mesh width of either interval may differ from $h$ and $\tilde{h}$.
} 
of a relatively small number of additional mesh points. For example, in the present set of experiments, when $\sigma=100$ there are 25 coarse grid points and 14 fine grid points in the horizontal direction; when $\sigma=1000$, there are 29 coarse and 43 fine grid points. (The unrefined mesh contains 31 points in each direction.) Table 4.7 shows the performance of the Gauss-Seidel and GMRES/ILU methods for four problems where mesh refinement is used, for the natural one-line ordering. Comparison with Table 4.1 shows that the behavior of the two iterative methods is essentially the same as that for uniform meshes. Similar conclusions apply for the three other ordering strategies. Thus, we conclude that the behavior on uniform meshes is indicative of behavior where mesh refinement is used to resolve boundary layers. (Experiments with the Gauss-Seidel method for $|\sigma|=|\tau|=1000$ were not performed because of storage constraints in our implementation.)

5. Experimental results: Separable variable coefficient problems. In this section, we examine the use of Corollary 2 to derive bounds on $\rho\left(D^{-1} C\right)$ when $A^{(b)}$ comes from a separable operator. We consider three model problems taken from [3]. Other experiments with these problems are described in [7].

PROBLEM 5.1.

$$
\begin{aligned}
-\Delta u+\frac{\sigma}{2}\left(1+x^{2}\right) u_{x}+\tau u_{y} & =0 & & \text { on } \Omega=(0,1) \times(0,1) \\
u & =0 & & \text { on } \partial \Omega .
\end{aligned}
$$

Discretization by centered differences gives, after scaling by $h^{2}$,

$$
\begin{gathered}
a_{i}^{(x)}=\alpha^{(x)}=a_{j}^{(y)}=\alpha^{(y)}=2, \\
c_{i+1} d_{i}=\left(1+\frac{\sigma h}{4}\left(1+x_{i+1}^{2}\right)\right)\left(1-\frac{\sigma h}{4}\left(1+x_{i}^{2}\right)\right) \\
\leq 1-\frac{1}{4}\left(\frac{\sigma h}{2}\right)^{2}+\sigma h^{2}=\xi, \\
b_{j+1} e_{j}=1-\left(\frac{\tau h}{2}\right)^{2}=\eta .
\end{gathered}
$$

For $\sigma \geq 0$ and $\tau \geq 0$, upwind discretization gives

$$
\begin{aligned}
a_{i} & =2+\frac{\sigma h}{2}\left(1+x_{i}^{2}\right) \geq 2+\frac{\sigma h}{2}=\alpha^{(x)}, \\
a_{j} & =2+\tau h=\alpha^{(y)}, \\
c_{i+1} d_{i} & =1+\frac{\sigma h}{2}\left(1+x_{i+1}^{2}\right) \leq 1+\sigma h=\xi, \\
b_{j+1} e_{j} & =1+\tau h=\eta .
\end{aligned}
$$


TABLE 5.1

Comparison of computed spectral radii and bounds for the block Gauss-Seidel iteration matrices, for Problem 5.1 with $h=1 / 32$.

\begin{tabular}{|c|c|c|c|c|c|c|c|c|}
\hline & \multicolumn{3}{|c|}{ Centered differences } & \multicolumn{4}{c|}{ Upwind differences } \\
\hline & \multicolumn{2}{|c|}{ One-line } & \multicolumn{2}{c|}{ Two-line } & \multicolumn{2}{c|}{ One-line } & \multicolumn{2}{c|}{ Two-line } \\
\hline$\sigma=\tau$ & Computed & Bound & Computed & Bound & Computed & Bound & Computed & Bound \\
\hline 20 & .741 & .809 & .674 & .731 & .817 & 1.298 & .772 & 1.379 \\
40 & .323 & .385 & .236 & .275 & .611 & 1.182 & .544 & 1.212 \\
60 & .047 & .062 & .015 & .018 & .455 & .961 & .386 & .985 \\
\hline
\end{tabular}

Table 5.1 compares the bounds for $\rho\left(\mathcal{L}_{1}\right)=\rho\left(D^{-1} C\right)^{2}$ obtained from Corollary 2 with the corresponding computed values of $\rho\left(\mathcal{L}_{1}\right)$, for $h=1 / 32$. For this problem, as well as the others considered below, we examine several choices of $\sigma$ and $\tau$ where for the largest such choice, $\max _{x_{i}}\left|r\left(x_{i}\right) h / 2\right|$ and $\max _{y_{j}}\left|s\left(y_{j}\right) h / 2\right|$ are both close to one.

PROBLEM 5.2.

$$
\begin{aligned}
-\Delta u+\sigma x^{2} u_{x} & =0 & & \text { on } \Omega=(0,1) \times(0,1), \\
u & =0 & & \text { on } \partial \Omega .
\end{aligned}
$$

Centered difference discretization gives

$$
\begin{aligned}
a_{i}^{(x)} & =\alpha^{(x)}=a_{j}^{(y)}=\alpha^{(y)}=2, \\
c_{i+1} d_{i} & =\left(1+\frac{\sigma h}{2} x_{i+1}^{2}\right)\left(1-\frac{\sigma h}{2} x_{i}^{2}\right) \leq 1+\frac{\sigma h^{2}}{2}-\frac{\sigma^{2} h^{4}}{2}=\xi, \\
b_{j+1} e_{j} & =1=\eta .
\end{aligned}
$$

Upwind difference discretization gives

$$
\begin{aligned}
a_{i} & =2+\sigma x_{i}^{2} h \geq 2+\sigma h^{3}=\alpha^{(x)}, \\
a_{j} & =2=\alpha^{(y)}, \\
c_{i+1} d_{i} & =1+\sigma x_{i+1}^{2} h \leq 1+\sigma h=\xi, \\
b_{j+1} e_{j} & =1=\eta .
\end{aligned}
$$

Table 5.2 compares bounds for $\rho\left(\mathcal{L}_{1}\right)$ with corresponding computed values for Problem 5.2. An entry "-" means that the analysis is not applicable because (3.11) is not satisfied.

TABLE 5.2

Comparison of computed spectral radii and bounds for the block Gauss-Seidel iteration matrices, for Problem 5.2 with $h=1 / 32$.

\begin{tabular}{|c|c|c|c|c|c|c|c|c|}
\hline & \multicolumn{3}{|c|}{ Centered differences } & \multicolumn{4}{c|}{ Upwind differences } \\
\hline & \multicolumn{2}{|c|}{ One-line } & \multicolumn{2}{c|}{ Two-line } & \multicolumn{2}{c|}{ One-line } & \multicolumn{2}{c|}{ Two-line } \\
\hline$\sigma=\tau$ & Computed & Bound & Computed & Bound & Computed & Bound & Computed & Bound \\
\hline 20 & .963 & 1.014 & .951 & .987 & .964 & 3.077 & .951 & 6.630 \\
40 & .953 & 1.033 & .939 & 1.011 & .955 & 10.37 & .939 & - \\
60 & .945 & 1.051 & .928 & 1.035 & .947 & 56.22 & .928 & - \\
\hline
\end{tabular}

\section{PROBLEM 5.3.}

$$
\begin{aligned}
-\Delta u+\sigma(1-2 x) u_{x}+\tau(1-2 y) u_{y} & =0 & & \text { on } \Omega=(0,1) \times(0,1), \\
u & =0 & & \text { on } \partial \Omega .
\end{aligned}
$$


Centered difference discretization gives

$$
\begin{aligned}
a_{i}^{(x)} & =\alpha^{(x)}=a_{j}^{(y)}=\alpha^{(y)}=2 \\
c_{i+1} d_{i} & =\left(1+\frac{\sigma h}{2}\left(1-2 x_{i+1}\right)\right)\left(1-\frac{\sigma h}{2}\left(1-2 x_{i}\right)\right) \\
& =1-2 h\left(\frac{\sigma h}{2}\right)-\left(\frac{\sigma h}{2}\right)^{2}\left(1-2 x_{i}\right)\left(1-2 x_{i+1}\right) \\
& \leq 1-\sigma h^{2}+\left(\frac{\sigma h}{2}\right)^{2}\left(2 h^{3}-h^{4}\right)=\xi \\
b_{j+1} e_{j} & =\left(1+\frac{\tau h}{2}\left(1-2 y_{j+1}\right)\right)\left(1-\frac{\tau h}{2}\left(1-2 y_{j}\right)\right) \\
& \leq 1-\tau h^{2}+\left(\frac{\tau h}{2}\right)^{2}\left(2 h^{3}-h^{4}\right)=\eta .
\end{aligned}
$$

For $\sigma \geq 0$ and $\tau \geq 0$, upwind discretization gives

$$
\begin{aligned}
a_{i} & =2+\sigma\left|1-2 x_{i}\right| h \geq 2=\alpha^{(x)}, \\
a_{j} & =2+\tau\left|1-2 y_{j}\right| h \geq 2=\alpha^{(y)}, \\
c_{i+1} d_{i} & =1+\sigma\left|1-2 x_{i+1}\right| h \leq 1+\sigma h=\xi, \\
b_{j+1} e_{j} & =1+\tau\left|1-2 y_{j+1}\right| h \leq 1+\tau h=\eta .
\end{aligned}
$$

Table 5.3 compares bounds and computed values of $\rho\left(\mathcal{L}_{1}\right)$ for Problem 5.3; the entry "-" indicates that either (3.9) or (3.11) is not satisfied.

TABLE 5.3

Comparison of computed spectral radii and bounds for the block Gauss-Seidel iteration matrices, for Problem 5.3 with $h=1 / 32$.

\begin{tabular}{|c|c|c|c|c|c|c|c|c|}
\hline & \multicolumn{3}{|c|}{ Centered differences } & \multicolumn{4}{c|}{ Upwind differences } \\
\hline & \multicolumn{2}{|c|}{ One-line } & \multicolumn{2}{c|}{ Two-line } & \multicolumn{2}{c|}{ One-line } & \multicolumn{3}{c|}{ Two-line } \\
\hline$\sigma=\tau$ & Computed & Bound & Computed & Bound & Computed & Bound & Computed & Bound \\
\hline 20 & .854 & .921 & .813 & .869 & .871 & 3.611 & .833 & 6.986 \\
40 & .733 & .852 & .669 & .785 & .780 & - & .723 & - \\
60 & .629 & .788 & .553 & .710 & .703 & - & .634 & - \\
\hline
\end{tabular}

To understand these results, it is useful to recall the constant coefficient problem (4.1). For that problem, the parameters associated with centered differences are given by (4.3). As shown in [7], [8], if both $\sigma h / 2<1$ and $\tau h / 2<1$, then the bounds from Corollary 2 essentially have the form $1-O\left(\sigma^{2} h^{2}\right)-O\left(\tau^{2} h^{2}\right)$. In particular, if either $\sigma h / 2$ or $\tau h / 2$ are near 1 , then $\xi$ or $\eta$ are close to 0 , and the bounds from Corollary 2 are very small. For Problem 5.1, $r(x)$ (the coefficient of $u_{x}$ ) is bounded below away from 0 , so that for large $\sigma$, the contribution $h r\left(x_{i}\right) / 2$ cannot be small for any $x_{i}$. Consequently, the bounding value $\xi$ is qualitatively like its constant coefficient counterpart (compare (5.1) and (4.3)). Moreover, $\alpha^{(x)}, \alpha^{(y)}$ and $\eta$ have the same values as in the constant coefficient case. (This is true for $\alpha^{(x)}$ and $\alpha^{(y)}$ with all three problems considered here.) Thus, the bounds from Corollary 2 behave like their constant coefficient analogues. For Problem 5.2, the upper bound $\xi$ corresponds to a value for $x_{i}(=h)$ for which the differential operator is locally nearly selfadjoint; the resulting bounds typically do not even guarantee convergence, and they are larger 
than what would be obtained in the selfadjoint case. For Problem 5.3, $\xi=1-O\left(\sigma h^{2}\right)$ and $\eta=1-O\left(\sigma h^{2}\right)$, which lead to asymptotic bounds of the form $1-O\left(\sigma h^{2}\right)-O\left(\tau h^{2}\right)$; these are larger than those occurring for Problem 5.1 but smaller than for Problem 5.2. Note that for all three problems, the bounding values are qualitatively similar to the behavior of $\mathcal{L}_{1}$.

The parameters for upwind differences applied to the constant coefficient problem are

$$
a_{i}^{(x)}=\alpha^{(x)}=2+\sigma h, \quad a_{j}^{(y)}=\alpha^{(y)}=2+\tau h, \quad \xi=1+\sigma h, \quad \eta=1+\tau h .
$$

In this case, the bounds on $\rho\left(D^{-1} C\right)$ from Corollary 2 are less than one, and they decrease with increasing $\sigma$ or $\tau$ (see [7], [8]). However, the extra inequalities required to define $\alpha^{(x)}$ and $\alpha^{(y)}$ decrease the size of the denominators in (3.10) and (3.12) and limit the usefulness of the corollary. For Problem 5.1, $\sigma h$ is replaced by $\sigma h / 2$ in $\alpha^{(x)}$, and the bounds on $\rho\left(D^{-1} C\right)$ are less than one only when $\sigma h$ is large. The bounds for Problems 5.2 and 5.3, where they are defined, do not provide any useful information.

6. Experimental results: Nonseparable variable coefficient problems. We now examine the performance of the iterative methods for solving some nonseparable problems. Our goals are to examine the effectiveness of the block Gauss-Seidel and SOR methods, and ILU-preconditioned GMRES, for solving such problems; and to determine whether the analytic results of [7], [8], and $\S 3$ are of use in predicting behavior.

The following problem, from [19], models the circular flow of a cold fluid with a hot wall at the right boundary.

PROBLEM 6.1.

$$
\begin{aligned}
-\epsilon \Delta u+2 y\left(1-x^{2}\right) u_{x}-2 x\left(1-y^{2}\right) u_{y} & =0 & & \text { on } \Omega=(-1,1) \times(0,1) \\
u & =0 & & \text { on } 0 \leq y \leq 1, x=-1 \\
u & =100 & & \text { on } 0 \leq y \leq 1, x=1 \\
u & =0 & & \text { on }-1 \leq x<0, y=0 \\
u_{n} & =0 & & \text { on } 0 \leq x \leq 1, y=0 \\
u & =0 & & \text { on }-1 \leq x \leq 1, y=1 .
\end{aligned}
$$

The velocity vectors have turning points in the vertical component, and their magnitudes vary throughout the domain of definition. The solution contains a boundary layer at $x=1$. Figure 6.1 shows the boundary conditions and streamlines, and the general shape of the solution, for $\epsilon=1 / 100 .^{3}$ A related problem, differing from Problem 6.1 only in the boundary conditions, was also considered in [9]; experimental results were qualitatively similar to those presented below.

As above, we consider both centered difference and upwind difference discretizations. At the outflow boundary $x \geq 0, y=0$, we used first order upwind differences

$$
0=u_{n}\left(x_{i}, 0\right)=u_{y}\left(x_{i}, y_{0}\right) \approx \frac{u\left(x_{i}, y_{1}\right)-u\left(x_{i}, y_{0}\right)}{h}
$$

i.e., $u\left(x_{i}, 0\right)=u\left(x_{i}, y_{1}\right)$. For the centered difference scheme, we consider both a square $31 \times 31$ mesh, and a uniform mesh of width $h=1 / 32$. The first choice

3 The discrete solution was computed using centered differences with 31 interior grid points in each direction; the figure includes the exact solution values at $x= \pm 1$ and $y=1$, but not at $y=0$. 

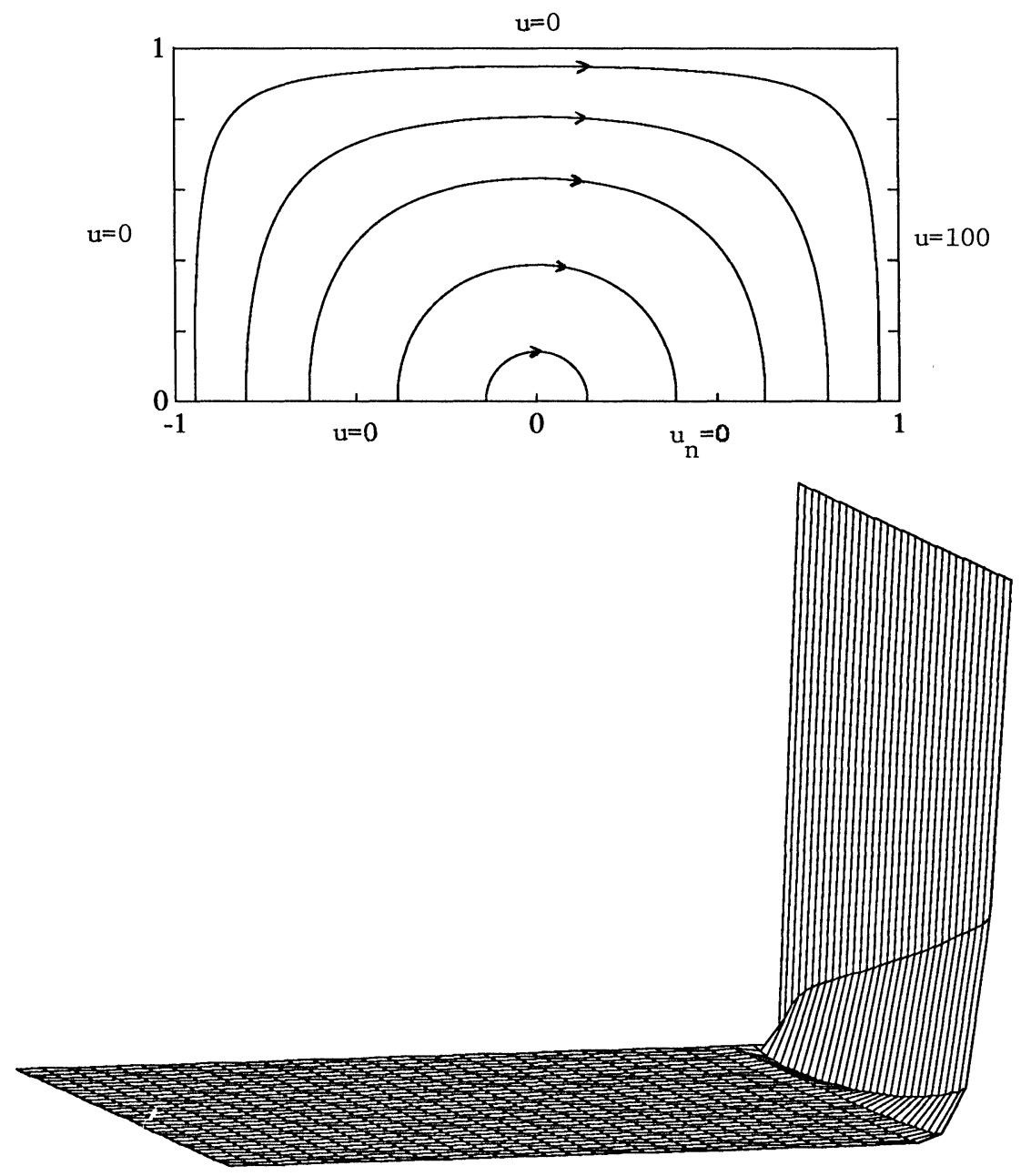

Fig. 6.1. Boundary conditions and solution for Problem 6.1.

produces matrices with the same algebraic structure as those considered in $\S \S 4-5$, but the horizontal mesh width is twice that of the vertical width; the second choice leads to lines of different length in the grid. We also consider a strategy for improving the accuracy of the solution, based on defect correction methods. For all tests, the initial guesses and stopping criteria are as in $§ 4$.

Table 6.1 shows average iteration counts for solving the reduced system derived when centered differences are applied on a square $31 \times 31$ grid. Here, the grid sizes for the full system are uniform in each of the $x$ and $y$ coordinates, with $h_{x}=1 / 16$ and $h_{y}=1 / 32$. As in the constant coefficient case (§4), block relaxation is most effective for intermediate values of $\epsilon^{-1}$, where it is competitive with GMRES/ILU. The latter method is more effective when $\epsilon^{-1}$ is either small or large. The performance of the stationary methods is fairly insensitive to the choice of ordering. This is consistent with the fact that, because of variable directions of flow, there is no clear correspondence between lines and flow direction. On the other hand, as in $\S 4$, the performance of GMRES/ILU is typically better with the natural orderings than with the red-black orderings. We remark that in a few experiments with Orthomin [5], we 


\section{TABLE 6.1}

Average iteration counts for Problem 6.1 on a $31 \times 31$ grid $\left(h_{x}=1 / 16, h_{y}=1 / 32\right)$, with centered differences. Numbers in parentheses are approximate number of digits of accuracy when methods did not meet the stopping criterion.

\begin{tabular}{|c|l|r|r|r|r|r|c|}
\hline \multicolumn{2}{|c|}{} & \multicolumn{1}{|c|}{$1 / \epsilon$} \\
\hline & Ordering & 10 & 50 & 100 & 200 & 500 & 1000 \\
\hline \multirow{3}{*}{ Gauss- } & Natural one-line & 122 & 22 & 27 & 57 & $150(4)$ & $150(1)$ \\
Seidel & Red-black one-line & 119 & 26 & 29 & 63 & $150(4)$ & $150(1)$ \\
& Natural two-line & 114 & 24 & 26 & 54 & $150(4)$ & $150(1)$ \\
& Red-black two-line & 111 & 25 & 26 & 54 & $150(4)$ & $150(1)$ \\
\hline \multirow{3}{*}{ GMRES } & Natural one-line & 10 & 7 & 7 & 8 & 15 & 33 \\
/ ILU & Red-black one-line & 27 & 27 & 34 & 37 & 46 & 74 \\
& Natural two-line & 14 & 10 & 10 & 10 & 19 & 87 \\
& Red-black two-line & 24 & 21 & 26 & 26 & 42 & 71 \\
\hline
\end{tabular}

found Orthomin(5) to be somewhat less robust than GMRES(5).

TABLE 6.2

Average iteration counts for Problem 6.1, with the natural one-line and two-line orderings, on a uniform grid with mesh size $h=1 / 32$ and centered differences. Numbers in parentheses are approximate number of digits of accuracy when methods did not meet the stopping criterion.

\begin{tabular}{|l|l|l|l|l|l|l|}
\hline & \multicolumn{7}{|c|}{$1 / \epsilon$} \\
\hline Method & 10 & 50 & 100 & 200 & 500 & 1000 \\
\hline G.S. Natural one-line & $150(5)$ & 28 & 22 & 35 & 122 & $150(3)$ \\
G.S. Natural two-line & 129 & 27 & 22 & 34 & 101 & $150(3)$ \\
GMRES/ILU Natural one-line & 17 & 11 & 10 & 10 & 16 & $150(3)$ \\
GMRES/ILU Natural two-line & 20 & 14 & 12 & 12 & 17 & 64 \\
\hline
\end{tabular}

Table 6.2 shows iteration counts for solving the reduced system derived from an underlying uniform mesh of width $h=1 / 32$, for block Gauss-Seidel and GMRES/ILU, with the two natural line orderings. The lines are oriented as in Fig. 2.2. These results are similar to those of Table 6.1, except that GMRES/ILU has trouble with one problem class $(\epsilon=1 / 1000$ with the natural one-line ordering). In this case, the iteration "stagnates," in the sense that the residual norm $\left\|g^{(b)}-A^{(b)} u_{i}^{(b)}\right\|_{2}$ remains constant over many iterations. ${ }^{4}$ In contrast, whenever the block relaxation methods fail to meet the stopping criterion, they appear to be converging.

\section{TABLE 6.3}

Average iteration counts for Problem 6.1 on a $31 \times 31$ grid $\left(h_{x}=1 / 16, h_{y}=1 / 32\right)$, with upwind differences.

\begin{tabular}{|c|l|r|r|r|r|r|c|}
\hline & \multicolumn{1}{|c|}{ Ordering } & 10 & 50 & 100 & 200 & 500 & 1000 \\
\hline & Natural one-line & 142 & 31 & 24 & 21 & 18 & 17 \\
Gauss- & Red-black one-line & 139 & 37 & 29 & 26 & 24 & 23 \\
Seidel & Natural two-line & 132 & 32 & 25 & 23 & 20 & 19 \\
& Red-black two-line & 131 & 35 & 27 & 22 & 20 & 19 \\
\hline \multirow{3}{*}{ GMRES } & Natural one-line & 10 & 8 & 8 & 7 & 7 & 6 \\
/ ILU & Red-black one-line & 29 & 25 & 28 & 32 & 36 & 37 \\
& Natural two-line & 15 & 10 & 10 & 9 & 8 & 7 \\
& Red-black two-line & 28 & 20 & 20 & 21 & 26 & 25 \\
\hline
\end{tabular}

Table 6.3 shows average iteration counts for solving the reduced system derived when upwind differences are applied to Problem 6.1. Note that the mesh points used

${ }^{4}$ Stagnation of this type also occurs for GMRES(10) and GMRES(15). 
for discretization depend on the direction of flow (see $\S 2$ ), and the reduced matrices $A^{(b)}$ are always diagonally dominant. The results of Table 6.3 (for the stationary methods) are consistent with those for constant coefficient problems.

A methodology for improving accuracy that does not require a priori knowledge about the solution is the class of defect correction methods. A description of this approach can be found in [12], which contains several other references. For the operator $L_{\epsilon} u \equiv-\epsilon \Delta u+r u_{x}+s u_{y}$, let $A_{\epsilon, h}$ denote the matrix associated with the (second order) centered difference discretization on a uniform mesh of width $h$. For $\hat{\epsilon}>\epsilon$, let $A_{\hat{\epsilon}, h}$ denote the analogous matrix derived from $L_{\hat{\epsilon}}$. In its simplest form, the defect correction iteration consists of the following steps, where $f$ is the discrete right-hand side.

Solve $A_{\hat{\epsilon}, h} u^{(m)}=f$.

For $m=0,1, \cdots$, Do

$$
\begin{aligned}
& r^{(m)}=f-A_{\epsilon, h} u^{(m)} \\
& \text { Solve } A_{\hat{\epsilon}, h} d^{(m)}=r^{(m)} \\
& u^{(m+1)}=u^{(m)}+d^{(m)}
\end{aligned}
$$

End

The idea is to compensate for instabilities associated with high order operators using lower order operators. For the choice $\hat{\epsilon}=\epsilon+c h$ where $c>0$ is a fixed constant, $A_{\hat{\epsilon}, h}$ is a first order discretization. At every step of the iteration, $A_{\epsilon, h}$ is used only to calculate the residual, and a linear system with coefficient matrix $A_{\hat{\epsilon}, h}$ must be solved. Thus, the cost of this method is highly dependent on the cost of solving the linear system.

Any $c>0$ prevents the convection terms from dominating the discrete problem, for arbitrarily small $\epsilon$. For $c \geq \max \{|r(x, y)| / 2,|s(x, y)| / 2\}, A_{\hat{\epsilon}, h}$ and the resulting reduced matrix $A_{\hat{\epsilon}, h}^{(b)}$ are diagonally dominant $M$-matrices. For Problem 6.1, this gives $c=1$. However, Hemker [15] has observed that (using a variant of the algorithm above) better accuracy is obtained with smaller $c$. Following [15], we use $c=\frac{1}{2}$. The differential operator $L_{\hat{\epsilon}}$ for Problem 6.1 is then equivalent to

$$
-\Delta u+\frac{2 y\left(1-x^{2}\right)}{\epsilon+h / 2} u_{x}-\frac{2 x\left(1-y^{2}\right)}{\epsilon+h / 2} u_{y} .
$$

We refer to the discretization of this operator by centered difference as the "defect correction discretization." Table 6.4 shows the performance of the various iterative methods for solving the resulting reduced linear systems. (See [15] for a discussion of the overall iteration.) These results are qualitatively similar to performance for upwind differences.

7. Concluding remarks. In this paper, we have continued the study of line iterative methods for solving reduced systems begun in [7], [8]. We have extended the analysis in two ways. First, for matrices that arise from variable coefficient separable differential operators, we derived conditions under which the reduced matrices can be symmetrized via diagonal similarity transformations; previous results applied only to constant coefficient problems. Symmetrization is the key to the analysis of convergence behavior for the constant coefficient case. In the present analysis, it determines conditions under which the classical analysis of SOR applies, from which the optimal SOR parameter can be expressed as a simple function of the maximum eigenvalue of the line Jacobi iteration matrix, and it leads to some analytic bounds on performance for separable problems. In addition, we used regular splitting results to show that the 
TABLE 6.4

Average iteration counts to solve the linear systems arising from the defect correction method, for the natural one-line and two-line orderings on a uniform grid with mesh size $h=1 / 32$. Numbers in parentheses are approximate number of digits of accuracy when methods did not meet the stopping criterion.

\begin{tabular}{|l|c|l|l|l|l|l|}
\hline & \multicolumn{7}{|c|}{$1 / \epsilon$} \\
\hline Method & 10 & 50 & 100 & 200 & 500 & 1000 \\
\hline G.S. Natural one-line & $150(4)$ & 42 & 32 & 28 & 26 & 25 \\
G.S. Natural two-line & $150(5)$ & 38 & 30 & 27 & 25 & 25 \\
GMRES/ILU Natural one-line & 17 & 12 & 12 & 11 & 11 & 11 \\
GMRES/ILU Natural two-line & 21 & 16 & 14 & 14 & 13 & 13 \\
\hline
\end{tabular}

analysis of line Jacobi splittings can be extended to splittings based on incomplete LU factorizations, for various line orderings of the reduced grid. The results help explain the good performance of IC preconditioners applied to the nonsymmetric matrix problems arising from the convection-diffusion equation.

We have also performed an extensive set of numerical experiments that examine the effects of direction of flow, discretization, and grid ordering on performance of the line iterative methods. For constant coefficient problems, the results reveal correlations between relaxation sweep direction and direction of flow that are not displayed by any analytic results. They also show that for block relaxation methods, red-black orderings are less sensitive to flow directions than natural orderings, whereas for IC-preconditioned GMRES, convergence is faster for natural orderings than for red-black orderings. In addition, both block relaxation and IC preconditioned GMRES are effective for many problems where the analysis does not apply. In general, IC-preconditioned GMRES is more robust than block relaxation. Finally, experimental results for problems with variable coefficients or locally refined grids are largely consistent with analysis and experiments for constant coefficients and uniform grids.

\section{REFERENCES}

[1] C. C. AshCraft AND R. G. GRIMES, On vectorizing incomplete factorization and SSOR preconditioners, SIAM J. Sci. Statist. Comput., 9 (1988), pp. 122-151.

[2] R. BeAuwens, Factorization iterative methods, M-operators and H-operators, Numer. Math., 31 (1979), pp. 335-357, 1979.

[3] E. F. F. BotTA AND A. E. P. Veldman, On local relaxation methods and their application to convection-diffusion equations, J. Comput. Phys., 48 (1981), pp. 127-149.

[4] R. C. Y. CHIN AND T. A. MANTEUFFEL, An analysis of block successive overrelaxation for a class of matrices with complex spectra, SIAM J. Numer. Anal., 25 (1988), pp. 564-585.

[5] S. C. Eisenstat, H. C. Elman, AND M. H. Schultz, Variational iterative methods for nonsymmetric systems of linear equations, SIAM J. Numer. Anal., 20 (1983), pp. 345-357.

[6] H. C. ElmAN, Relaxed and stabilized incomplete factorizations for non-self-adjoint linear systems, BIT, 29 (1989), pp. 890-915.

[7] H. C. Elman AND G. H. GoluB, Iterative methods for cyclically reduced non-self-adjoint linear systems, Math. Comp., 54 (1990), pp. 671-700.

[8] — Iterative methods for cyclically reduced non-self-adjoint linear systems II, Report UMIACS-TR-89-45, Department of Computer Science, University of Maryland, College Park, MD, 1989; Math. Comp., 56 (1991), pp. 215-242.

[9] — Line iterative methods for cyclically reduced discrete convection-diffusion problems, Report UMIACS-TR-90-16, Department of Computer Science, University of Maryland, College Park, MD, 1990.

[10] K. FAN, Note on M-matrices, Quart. J. Math. Oxford Ser. (2), 11 (1960), pp. 43-49.

[11] G. E. Forsythe AND W. R. WASOW, Finite Difference Methods for Partial Differential Equations, John Wiley and Sons, New York, 1960.

[12] W. HackвUSCH, Multi-Grid Methods and Applications, Springer-Verlag, Berlin, 1985. 
[13] L. A. HAGEMAN AND R. S. VARGA, Block iterative methods for cyclically reduced matrix equations, Numer. Math., 6 (1964), pp. 106-119.

[14] L. A. Hageman And D. M. Young, Applied Iterative Methods, Academic Press, New York, 1981.

[15] P. W. HEMKER, Mixed defect correction iteration for the accurate solution of the convection diffusion equation, in Multi-grid Methods, W. Hackbusch and U. Trottenberg, eds., Lecture Notes in Mathematics 960, Springer-Verlag, Berlin, 1982.

[16] L. LAMPORT, The parallel execution of DO loops, Comm. ACM., 17 (1974), pp. 83-93.

[17] J. A. MEIJERINK AND H. A. VAN DER VORST, An iterative solution method for linear systems of which the coefficient matrix is a symmetric M-matrix, Math. Comp., 31 (1977), pp. 148162.

[18] J. P. Milaszewicz, Improving Jacobi and Gauss-Seidel iterations, Lin. Alg. Appl., 93 (1987), pp. $161-170$.

[19] K. W. MoRTon, Generalised Galerkin methods for steady and unsteady problems, in Numerical Methods for Fluid Dynamics, K. W. Morton and M. J. Baines, eds., Academic Press, Orlando, FL, 1982.

[20] S. V. PARTER AND M. STEUERWALT, Block iterative methods for elliptic and parabolic difference equations, SIAM J. Numer. Anal., 19 (1982), pp. 1173-1195.

[21] PCGPAK User's Guide, Version 1.04, Scientific Computing Associates, New Haven, CT, 1987.

[22] P. J. RoAche, Computational Fluid Dynamics, Hermosa Publishers, Albuquerque, 1982.

[23] Y. SAAD AND M. H. SChUlTZ, GMRES: A generalized minimual residual algorithm for solving nonsymmetric linear systems, SIAM J. Sci. Statist. Comput., 7 (1986), pp. 856-869.

[24] J. Sheldon, On the spectral norms of several iterative processes, J. Assoc. Comput. Mach., 6 (1959), pp. 494-505.

[25] R. S. VARGA, Matrix Iterative Analysis, Prentice-Hall, Englewood Cliffs, NJ, 1962.

[26] H. F. Weinberger, A First Course in Partial Differential Equations with Complex Variables and Transform Methods, Blaisdell, New York, 1965.

[27] Z. Woz̀NICKI, Two-sweep iterative methods for solving large linear systems and their application to the numerical solution of multi-group multi-dimensional neutron diffusion equation, Ph.D. thesis, Report $N^{0} 1447 /$ CYFRONET/PM/A, Institute of Nuclear Research, Swierk, Poland, 1973.

[28] D. M. Young, Iterative Solution of Large Linear Systems, Academic Press, New York, 1971. 